\title{
Modelling Material-Salt Interactions in Molten Salt Reactors: Opportunities and Path Forward
}

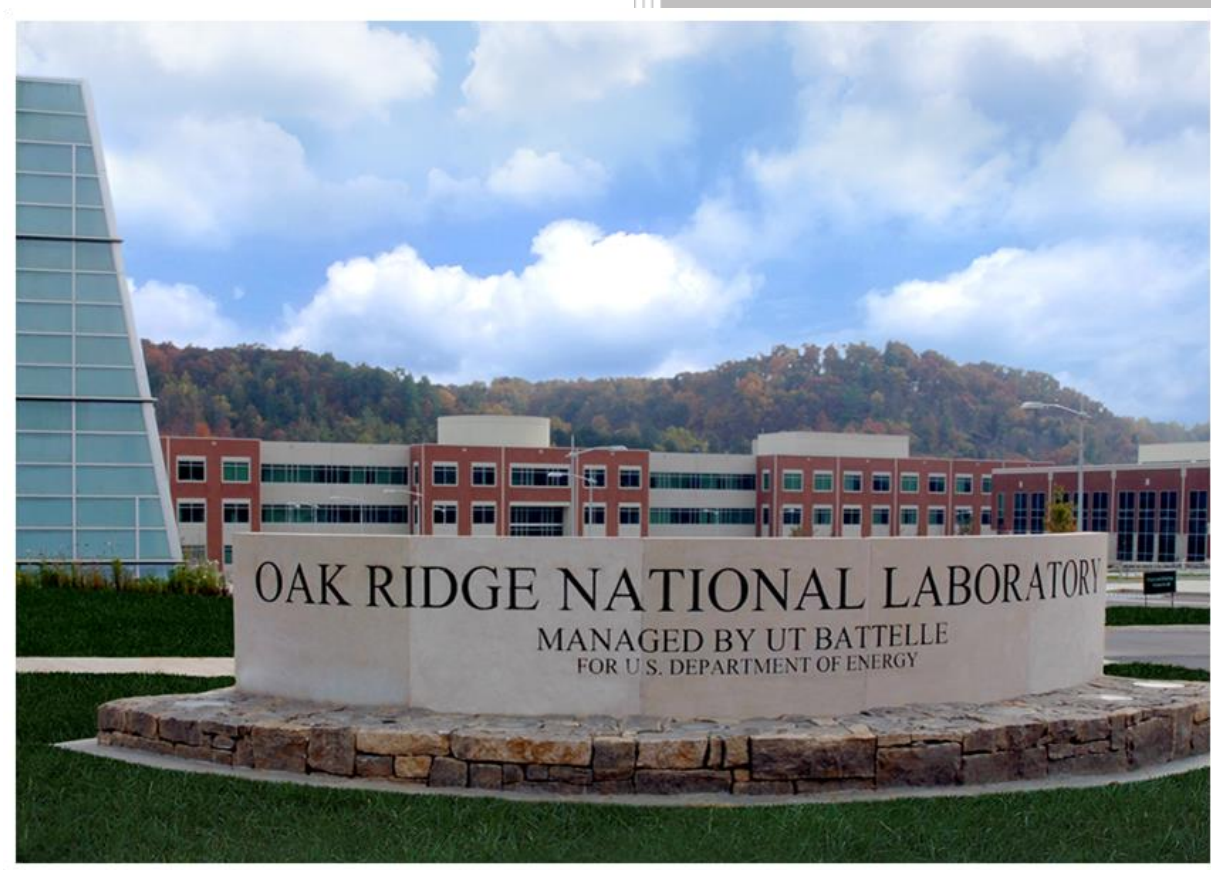

Approved for public release. Distribution is unlimited

J.K. Startt

C.S. Deo

S.S. Raiman

$5 / 30 / 2019$ 


\section{DOCUMENT AVAILABILITY}

Reports produced after January 1, 1996, are generally available free via US Department of Energy (DOE) SciTech Connect.

Website www.osti.gov

Reports produced before January 1, 1996, may be purchased by members of the public from the following source:

National Technical Information Service

5285 Port Royal Road

Springfield, VA 22161

Telephone 703-605-6000 (1-800-553-6847)

TDD 703-487-4639

Fax 703-605-6900

E-mailinfo@ntis.gov

Website http://classic.ntis.gov/

Reports are available to DOE employees, DOE contractors, Energy Technology Data Exchange representatives, and International Nuclear Information System representatives from the following source:

Office of Scientific and Technical Information

PO Box 62

Oak Ridge, TN 37831

Telephone 865-576-8401

Fax 865-576-5728

E-mail reports@osti.gov

Website http://www.osti.gov/contact.html

This report was prepared as an account of work sponsored by an agency of the United States Government. Neither the United States Government nor any agency thereof, nor any of their employees, makes any warranty, express or implied, or assumes any legal liability or responsibility for the accuracy, completeness, or usefulness of any information, apparatus, product, or process disclosed, or represents that its use would not infringe privately owned rights. Reference herein to any specific commercial product, process, or service by trade name, trademark, manufacturer, or otherwise, does not necessarily constitute or imply its endorsement, recommendation, or favoring by the United States Government or any agency thereof. The views and opinions of authors expressed herein do not necessarily state or reflect those of the United States Government or any agency thereof. 
Materials Science and Technology Division

Modelling Salt-Material Interactions in Molten Salt Reactors: Opportunities and Path Forward

\author{
Author(s) \\ Jacob K. Startt \\ Chaitanya S. Deo \\ Stephen S. Raiman
}

Date Published:

$5 / 30 / 2019$

\author{
Prepared by \\ OAK RIDGE NATIONAL LABORATORY \\ Oak Ridge, TN 37831-6283 \\ managed by \\ UT-BATTELLE, LLC \\ for the \\ US DEPARTMENT OF ENERGY \\ under contract DE-AC05-00OR22725
}





\section{CONTENTS}

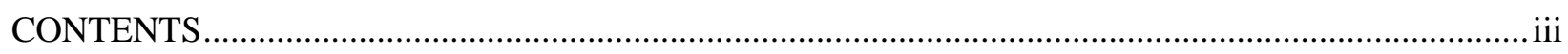

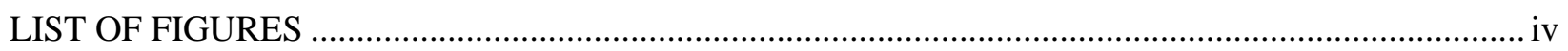

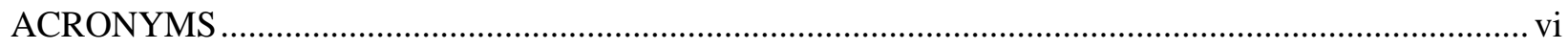

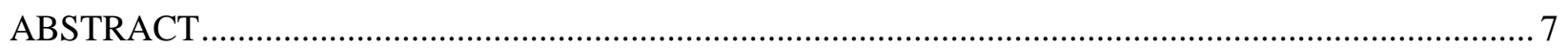

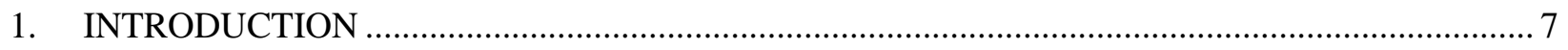

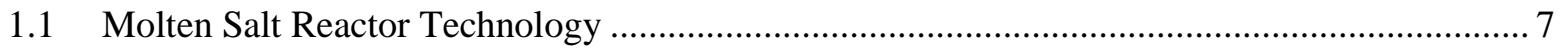

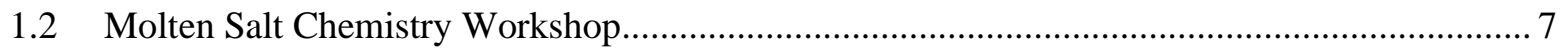

1.3 Opportunities for Computational Modelling................................................................... 8

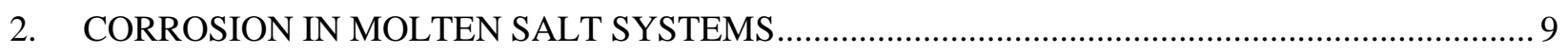

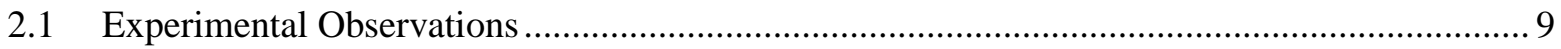

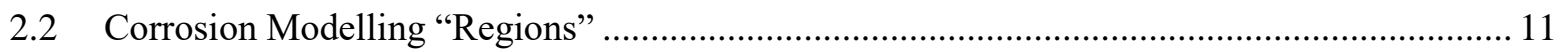

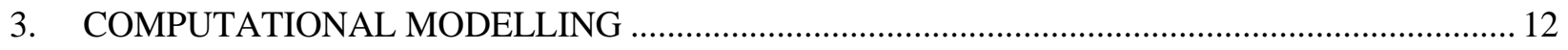

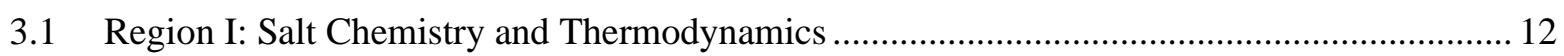

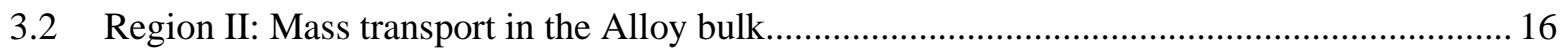

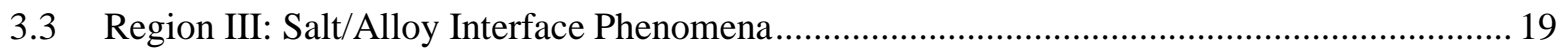

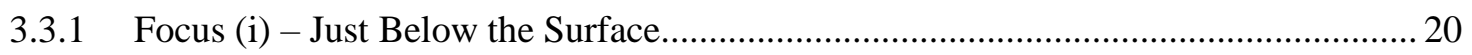

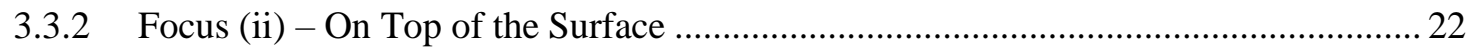

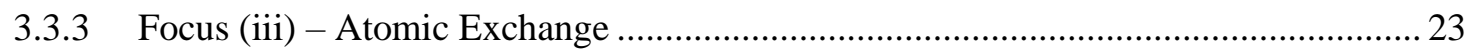

3.4 Connecting Regions with Non-Equilibrium Modelling .................................................... 25

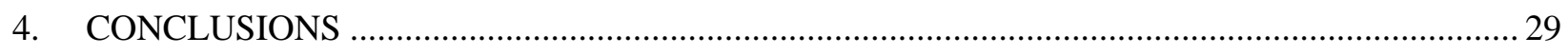

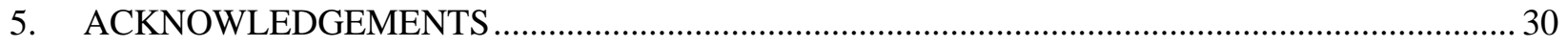

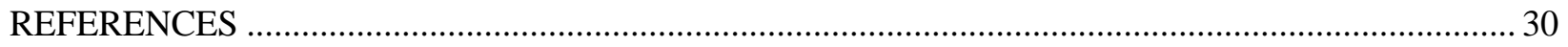




\section{LIST OF FIGURES}

Figure 1. Calculated ellingham diagram showing stability of metallic chlorides.

Figure 2. EDS maps depicting depth of Cr depletion in 316 Stainless steel corroded in a purified fluoride based $\mathrm{FLiBe}$ salt at a temperature of $700^{\circ} \mathrm{C}$.

Figure 3. SEM images (a,e) and EDS maps depicting $\mathrm{Cr}$ depletion and $\mathrm{O}, \mathrm{Mg}$ and $\mathrm{Na}$ impregnation in $\mathrm{Ni}$ alloy 230.

Figure 4. Illustration of a Cr containing alloy surface under a molten salt.

Figure 5. AIMD calculated self-diffusion coefficients for a $\mathrm{LiCl}-\mathrm{KCl}$ salt mixture.

Figure 6. AIMD calculated Gibbs free energy of mixing for eutectic $\mathrm{LiCl}-\mathrm{KCl}$ salt mixture at $823^{\circ} \mathrm{C}$

Figure 7. Pair distribution functions and coordination numbers calculated using IPMD methods for the $\mathrm{NaCl}-\mathrm{ZnCl}_{2}$ binary sub-system of $\mathrm{NaCl}-\mathrm{KCl}-\mathrm{ZnCl}_{2}$.

Figure 8. Enthalpies of formation calculated using IPMD for the liquid $\mathrm{NaCl}-\mathrm{KCl}-\mathrm{ZnCl}_{2}$ ternary salt at $827^{\circ} \mathrm{C}$.

Figure 9. Equilibrium phase diagrams from IPMD and CALPHAD calculations.

Figure 10. DFT NEB calculated vacancy migration energy paths in $\mathrm{Ni}_{3} \mathrm{Al}$.

Figure 11. Examples of atomic configurations of a Ni-Cr alloy that could be used to model $\mathrm{Cr}$ migration energies and paths with DFT NEB methods.

Figure 12. Surface slabs used to calculate surface segregation energies in fcc Ni.

Figure 13. Calculated surface segregation energies for several transition metals in an fcc Ni (111) surface.

Figure 14. Calculated adsorption energy of $\mathrm{S}$ on a $\mathrm{Co}(0001)$ surface from DFT.

Figure 15. AIMD simulation of U nanocluster on a Mo(110) surface under a vacuum.

Figure 16. AIMD simulation of $\mathrm{U}$ nanocluster on a $\mathrm{Mo}(110)$ surface under a $\mathrm{LiCl}-\mathrm{KCl}$ liquid salt.

Figure 17. DFT calculated dissolution (desorption) energies for several dissolution products for a $\mathrm{Cu}(111)$ as function of $\mathrm{Cl}$ surface coverage $(\theta)$.

Figure 18. AIMD simulation of the adsorption of a $\mathrm{CH}_{3}-\mathrm{Al}-\mathrm{CH}_{2}$ molecule on graphene. 25

Figure 19. Relative length- and timescales of several of the most commonly employed computational materials science models that can be incorporated into multi-scale modelling. 26

Figure 20. Different $\mathrm{NaCl}$ (100) surface structures used to model dissolution. 
Figure 21. Results of AIMD modelling of $\mathrm{NaCl}$ dissolution in Water.

Figure 22. kMC modelled dissolution of an $\mathrm{NaCl}$ nanocrystal in water............................................... 28

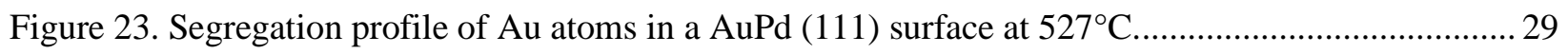




\section{ACRONYMS}

$\begin{array}{ll}\text { AIMD } & \text { ab-initio molecular dynamics } \\ \text { ARE } & \text { Aircraft Reactor Experiment } \\ \text { CALPHAD } & \text { CALculation of PHAse Diagrams } \\ \text { DFT } & \text { density functional theory } \\ \text { DOE } & \text { Department of Energy } \\ \text { FRD } & \text { Focused Research Direction } \\ \text { GIF } & \text { Generation IV International Forum } \\ \text { IP } & \text { interatomic potential } \\ \text { IPMD } & \text { interatomic potential molecular dynamics } \\ \text { kMC } & \text { kinetic Monte Carlo } \\ \text { MD } & \text { molecular dynamics } \\ \text { ML } & \text { Mono-layer } \\ \text { MOCVD } & \text { metal-organic chemical vapor deposition } \\ \text { MSR } & \text { molten salt reactor } \\ \text { MSRE } & \text { Molten Salt Reactor Experiment } \\ \text { NEB } & \text { nudged elastic band method } \\ \text { NN } & \text { Neural Network } \\ \text { ORNL } & \text { Oak Ridge National Laboratory } \\ \text { PDF } & \text { pair distribution function } \\ \text { XAFS } & \text { X-ray absorption fine-structure }\end{array}$




\begin{abstract}
A revitalized interest in molten salt reactor technology has resulted in a concerted effort across US based national laboratories and universities to investigate and overcome the remaining scientific and engineering questions facing the industry. This report provides a detailed description of the atomistic modelling techniques that could be employed to investigate the atomic-level forces and behaviors governing salt chemistry and thermodynamics, and the corrosion of container materials in these systems. The molten salt environment is broken down into three unique regions in which atomic behaviors are governed by separate processes and forces. A discussion is given on how each region can be modelled using different computational methods. The objective of the models presented for each region is to understand and quantify the individual atom-to-atom forces driving corrosion processes and changes in salt chemistry. Once these fundamental mechanisms are better understood, larger models, which connect the regions, can be designed to investigate the long-term non-equilibrium behaviors exhibited by these molten salt systems. With a large enough computational effort, employing the Modelling techniques discussed in this report to supplement experimental investigations, a greater understanding of the degradation of salt-facing structural materials can be gained, enabling more accurate lifetime prediction, greater safety and regulatory compliance, and faster material innovation.
\end{abstract}

\title{
1. INTRODUCTION
}

\subsection{MOLTEN SALT REACTOR TECHNOLOGY}

In 2002, shortly after its founding, the Generation IV International Forum (GIF) selected molten salt based nuclear reactors as one of the six most promising new designs for the next generation of advanced nuclear reactors [1]. These molten salt reactors (MSRs) could be defined as reactors in which the primary coolant loop is a molten salt. A variety of designs have been proposed, some of which are fast-reactor concepts, which typically employ a chloride salt, while others are thermal concepts, generally employing a fluoride salt and an in-core moderator. In most proposed designs, the coolant is also the fuel medium, with uranium or thorium fuel as part of the salt melt. Solid-fuel concepts have also been proposed [2]. A thorough discussion of proposed reactor types is available in [3]

Much of the existing database of knowledge for molten salt chemistry and technology was developed decades ago at Oak ridge National Laboratory (ORNL) during the Aircraft Reactor Experiment (ARE) in 1954 [4] and the Molten Salt Reactor Experiment (MSRE) operated from 1965 to 1969 [5]. These experiments, however, were narrow in scope and limited by the existing experimental methods available at the time and as such, there are sizable gaps in knowledge for these systems. Fortunately, the last several decades have seen large strides in both experimental methods and capabilities in addition to the advent of computational atomistic materials modelling, a tool that was not available to MSR researchers in the early $50 \mathrm{~s}$ and $60 \mathrm{~s}$.

\subsection{MOLTEN SALT CHEMISTRY WORKSHOP}

The renewed interest in MSR technologies, established by GIF, coupled with more advanced experimental tools and the newly available computational abilities of the current era has resulted in nearly two new decades worth of new research and collaboration between several Department of Energy (DOE) National US laboratories, research universities, and private sector companies. This effort culminated in 2017 when the DOE Office of Nuclear Energy hosted the Molten Salt Chemistry Workshop at ORNL to review the current level of MSR science and technology, and to establish a concerted research effort 
across US institutions to answer the most pressing science-based problems standing in the way of MSR technology deployment in industry [3].

At this workshop, six Future Research Directions (FRDs) for the advancement and development of MSR technologies were established. Each FRD was concentrated on a particular area of the MSR system and focused on establishing, for each area, the current level of understanding of material behaviors, the specific places where a better understanding is needed, and then the potential experimental and computational techniques that could be employed to achieve that better understanding. FRD 1 establishes the importance of understanding the physical and thermodynamic properties of the salt, such as structure, coordination, speciation and redox states, and then discusses the available experimental and computational methods available to study them. FRD 2 highlights the recent advances in experimental methods for determining these properties that were not previously available to ORNL researchers during the original MSR experiments, with a particular emphasis on diffraction and spectroscopic techniques. The third FRD extends the discussion of salt chemistry in FRD's 1 and 2 to salt radiochemistry and salt behavior in the presence of fission and activation products. Understanding the solubility, insolubility, volatility, and corrosiveness of these products in an MSR system is critically important for the prediction of long-term behavior in molten nuclear salt systems. The fourth FRD targets the reactions and interactions taking place at the salt and container interface. A particular focus is given to the elucidation of corrosion related processes through both experimental and computational methods. The last two FRDs aim to combine the insights and knowledge learned through FRDs 1-4 to develop new materials for use in MSR technologies (FRD 5) and to create a fully encompassing virtual reactor simulation to predict and model lifetime behaviors on par with models for the current generation of pressurized water reactors (FRD 6).

\subsection{OPPORTUNITIES FOR COMPUTATIONAL MODELLING}

The workshop's FRDs effectively serve as a blueprint for the future of US based materials-focused research and development in MSR technology, while also establishing salt chemistry and corrosion processes as the primary engineering related areas of focus that must be better understood before industrial deployment can be achieved. These processes and properties, however, often need to be studied in-situ and due to the intrinsic properties of molten salt systems, such as high temperatures, corrosiveness and volatility, these experimental efforts can quickly become difficult, cumbersome, expensive and in some cases impossible. As emphasized and discussed at the Workshop, it is evident that carefully devised computational modelling could serve to mitigate some of the problems facing experimental investigation and could potentially reduce the amount of work necessary to investigate and develop new materials.

The goal of this report is therefore to expand on the role of computational modelling efforts discussed at the Molten Salt Workshop and to provide an in-depth technical review of the specific atomistic based models relevant to molten salt chemistry. As corrosion related processes are at the core of both salt chemistry and interfacial behavior, a particular emphasis is placed on models and methods that could lead to new knowledge about the corrosion related mechanisms and processes in molten salt systems.

In the next section, a brief review of the current level of understanding of MSR corrosion processes and how that governs the choice and design of computational models is presented. The corrosion "problem" is then divided into three distinct environments, each corresponding to a physical region associated with the salt/metal interaction and which is governed by unique atomic-level forces and processes. The sections that follow, then describe the specific computational techniques and methods appropriate for modelling properties and behaviors in each of the three regions. When appropriate, brief literature reviews and examples of relevant modelling techniques and calculations previously performed for similar material systems are provided and discussed in terms of accuracy, reliability, and usefulness. 


\section{CORROSION IN MOLTEN SALT SYSTEMS}

\subsection{EXPERIMENTAL OBSERVATIONS}

The primary mode of alloy degradation in molten salt corrosion is the selective depletion of the most active alloying elements. Most high-temperature structural alloys are Ni-based alloys such as Alloys $600,617,625, \mathrm{C} 276,230,282$, or 740. Some Fe-based alloys are capable of service at temperatures above $600^{\circ} \mathrm{C}$, such as $316 \mathrm{H}$ and $800 \mathrm{H}$. For most common high-temperature engineering alloys, whether Ni or $\mathrm{Fe}$ based, $\mathrm{Cr}$ is the most active major alloy element. Therefore, depletion or dissolution of $\mathrm{Cr}$ atoms near the salt-facing surface and along grain boundaries connected to the surface is the primary concern. Figure 1 shows an Ellingham diagram of common metallic chlorides. $\mathrm{CrCl}_{2}$ is more stable than the chlorides formed with $\mathrm{Fe}, \mathrm{Ni}$, and $\mathrm{Mo}$, and therefore it is the first major alloying element to deplete in most high temperature alloys.

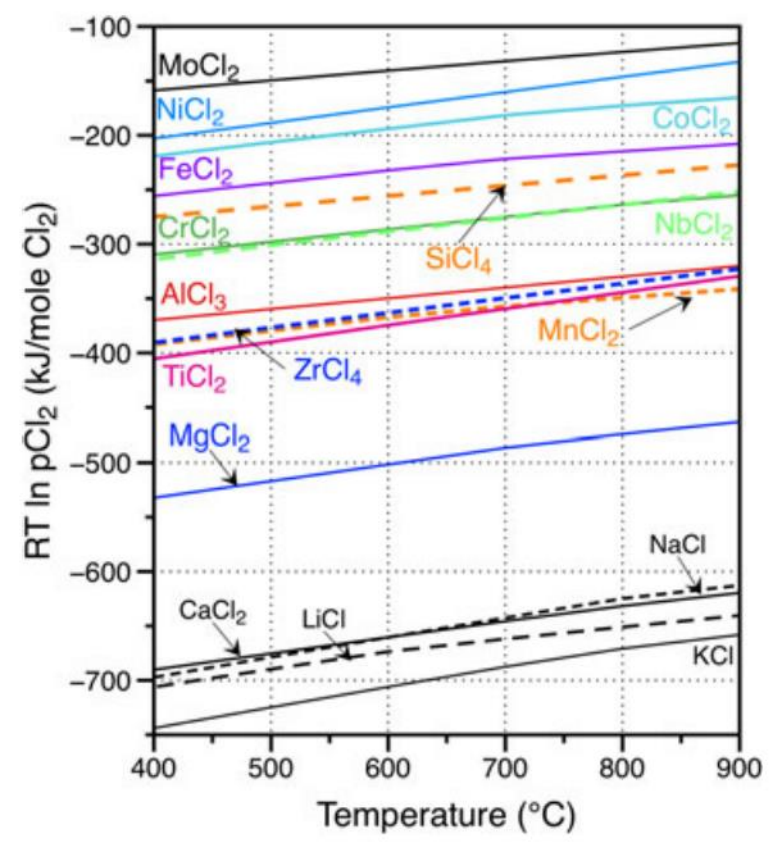

Figure 1. Calculated Ellingham diagram showing stability of metallic chlorides. Figure reproduced from Pint et al. [6]

This phenomenon can be seen in Figure 2 and Figure 3, which depict past experimental studies on Cr depletion depths in 316 stainless steel corroded under a FLiBe salt and Alloy 230 corroded under a $\mathrm{K}-\mathrm{Mg}-\mathrm{Na}$ chloride salts, respectively. Figure $3(\mathbf{c}, \mathbf{d}, \mathbf{g}, \mathbf{h})$ also shows the tendency and depth to which salt constituents and impurities can penetrate into the surface of the alloys $[6,7]$.

This exchange of atomic constituents at the salt/alloy interface also demonstrates the correlation between corrosion effects and overall salt chemistry. As alloy constituents leech into the salt, the salt chemistry will undoubtedly change, which will in response alter corrosion rates and mechanisms and so 
on until an equilibrium is reached or until either the alloy structurally fails or the salt loses its functionality.
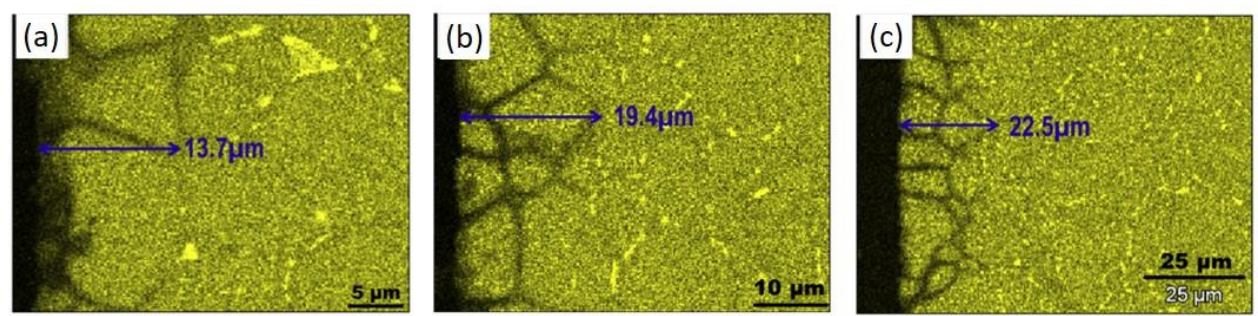

Figure 2. EDS maps depicting depth of $\mathrm{Cr}$ depletion in 316 Stainless steel corroded in a purified fluoride based FLiBe salt at a temperature of $700^{\circ} \mathrm{C}$ for (a) $1000 \mathrm{~h}$, (b) $2000 \mathrm{~h}$ and (c) $3000 \mathrm{~h}$. Figure reproduced from Zheng et al. [7].
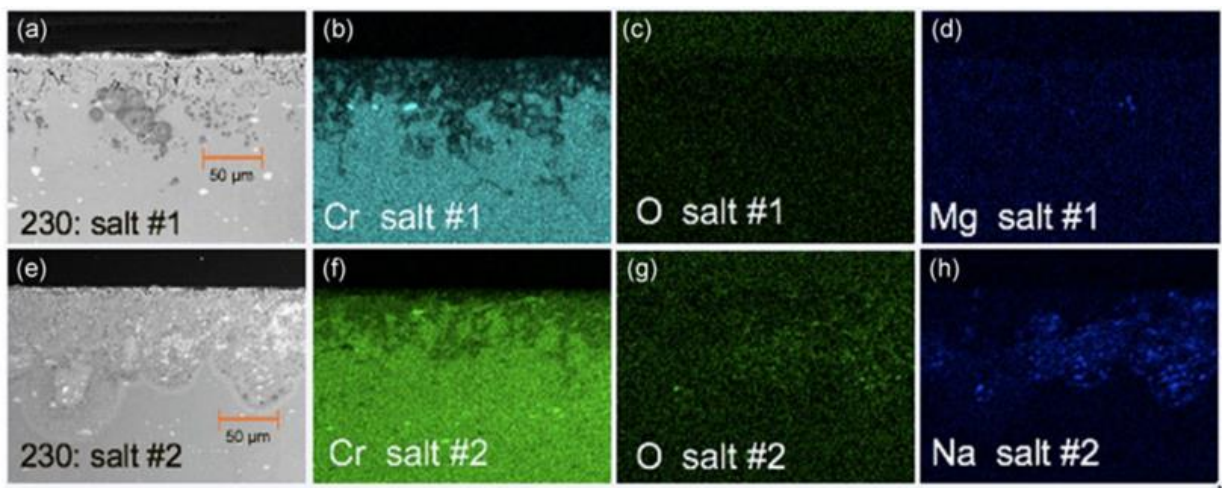

Figure 3. SEM images (a,e) and EDS maps (b-d, f-h) depicting Cr depletion and $\mathrm{O}, \mathrm{Mg}$ and $\mathrm{Na}$ impregnation in Ni alloy 230 corroded under two purities of a commercial $\mathrm{K}-\mathrm{Mg}-\mathrm{Na}$ chloride salt for $100 \mathrm{~h}$ at $800^{\circ} \mathrm{C}$. Figure reproduced from Pint et al. [6].

Models designed to calculate properties and processes associated with molten salt chemistry and corrosion phenomena should initially be focused on the fundamental physics and mechanisms driving the exchange and transport of atoms between the salt and alloy. Then, once the driving forces and exchange mechanisms are better understood, larger, longer and more extensive Modelling methods can be designed to simulate overall corrosion and salt behavior for time lengths relevant to an engineering or reactor lifetime scale. 


\subsection{CORROSION MODELLING "REGIONS"}

When designing models to study these fundamental corrosion forces and behaviors, it is useful to picture the entire corrosion process in terms of three separate regions:

I. The bulk salt

II. The bulk alloy

III. The salt-alloy interface

The distinction between each of the regions can be made in the types of unique local atomic interactions and forces occurring that are likely driving or contributing to the overall corrosion process. A diagram depicting the designation of these regions for a salt/alloy interface is given in Figure 3 .

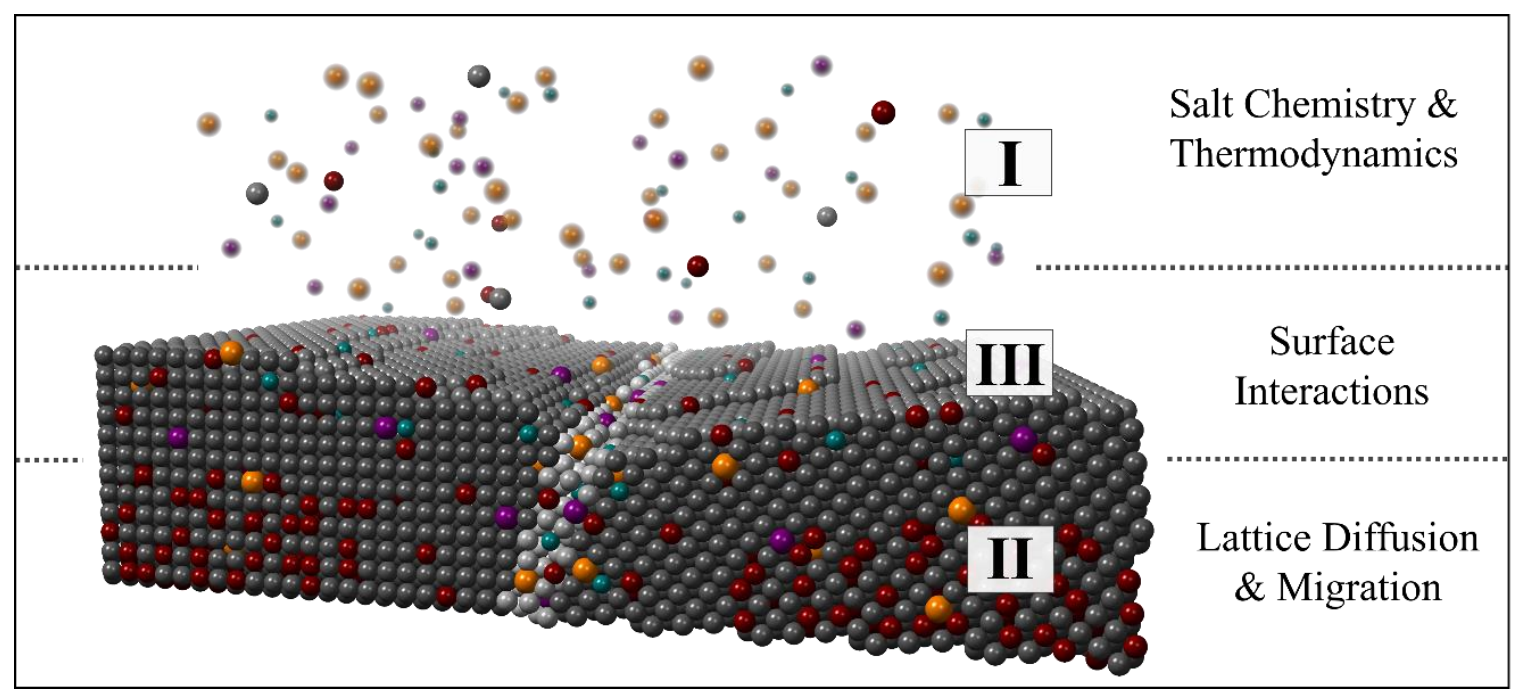

Figure 4. Illustration of a $\mathrm{Cr}$ (red) containing alloy surface under a molten salt. The picture is divided into three sections to represent regions in which the atomic level corrosion effects and mechanisms should be governed by unique physics and interactions. Region I represents the molten salt, governed by the concentrations of salt, alloy, and impurity constituents. Region II represents the bulk alloy just below the surface, governed by the diffusion and migration of both alloy and salt constituents and other defects through the atomic lattice. Region III represents the salt/alloy interface, governed by the local salt interactions and reactions with the alloy surface atoms.

The molten salt should be considered Region I, where salt chemistry and thermodynamics are the primary focus. In this region, changes in the salt phase behavior will be driven by temperature fluctuations and concentration changes as degraded alloy components and fission products enter and accumulate in the salt. Changes in salt speciation, coordination, and phase stability as well as alloy and fission product solubilities can be directly modelled with ab-initio molecular dynamics (AIMD) computational models. Additionally, several other important properties can be evaluated with these methods such as melting points, density, viscosity, heat capacity, thermal conductivity, vapor pressure, heat of fusion, expansivity, compressibility, and surface tension.

Region II encompasses the bulk alloy region just below the surface and is governed by the diffusion of atoms and defects through the atomic lattice and along grain boundaries. Density functional 
theory (DFT) and AIMD methods can be used to explore the driving forces that facilitate both $\mathrm{Cr}$ (or other alloy component) diffusion from the bulk and salt penetration deep into the alloy. Migration and diffusion of alloy and salt components along grain boundaries and the formation of voids, clusters and other defects can also be considered a part of this this region.

The actual interface between the molten salt and alloy surface makes up Region III. In this region, the specific forces and interactions between the salt ions and the surface atoms of the alloy (and other surface features like defects, dislocations and grain boundaries) is of interest. This is where the core of the currently unknown fundamental corrosion mechanisms can be thought to reside. Carefully devised DFT and AIMD simulations can be used to elucidate the forces that might be driving these corrosion mechanisms and processes.

Properties and features native to each region are capable of being modelled independently of one another using localized atomic system simulation cells that are able to circumvent the overall nonequilibrium character of the molten salt environment. For example, ab-initio methods can be used to study the local atomic driving forces of $\mathrm{Cr}$ depletion, a largely non-equilibrium process that is likely dependent on different atomic behaviors in all three regions, by simulating the near instantaneous forces on a $\mathrm{Cr}$ atom due to its local environment and coordination shell. By altering the coordination and local neighborhood of atoms around the $\mathrm{Cr}$ atom, fundamental diffusion and migration forces can be learned. Once a solid grasp on these basic driving forces and fundamental interactions is achieved, larger models that combine dependent processes across multiple regions can be used to investigate non-equilibrium effects on the diffusion of $\mathrm{Cr}$ (or other) atoms through the bulk or along the surface. These secondary models would likely involve combining ab-initio and molecular dynamics (MD) model data with multiscale and rate-dependent kinetic Monte Carlo (kMC) methods, which could allow for larger atomic system sizes and longer simulation times than the more computationally expensive ab-initio methods.

In the following sections, the models and simulations capable of investigating the physical and chemical processes present in each of the three regions are presented and discussed. Examples of similar or relevant computational investigations are referenced from already existing literature to support the modelling methods proposed for each region. Lastly, an overview of the potential kMC models that could be used to model non-equilibrium molten salt corrosion and salt chemistry environments will be discussed in terms of their accuracy and precision and in terms of the data and parameter requirements necessary to construct them.

\section{COMPUTATIONAL MODELLING}

\subsection{REGION I: SALT CHEMISTRY AND THERMODYNAMICS}

A full understanding of the chemical and thermodynamic behavior of the salt in its molten state is necessary not just for understanding properties related to corrosion but also to allow for accurate predictions of reactor system lifetime behaviors. Many of the questions at the forefront of salt chemistry are related to phase behavior and salt speciation. These are important properties that need to be understood because they directly dictate the solubility of contaminants in the liquid salt such as fission products or corroded alloy components. Composition changes (from further corrosion or fission) and temperature variations inside the coolant and fuel loops can result in increased corrosion rates, material deposition at the alloy interface, and gaseous bubble formation.

An understanding of these behaviors for a material system can be directly learned from its equilibrium phase diagram. This, however, poses a problem for the MSR community, as many of the 
binary and ternary phase diagrams needed for the liquid salt systems under investigation have never been constructed. The primary method for phase diagram construction is the CALPHAD method, which can be used to model molten salt systems [8-10]. In this approach, experimentally measured and/or first principles-derived thermodynamic and thermophysical properties of all phases in a material system are used to empirically fit models to construct a thermodynamic database to predict the most stable phase(s) under different temperature, composition, and pressure conditions. For a solution phase, the model Gibbs free energy is fit to data such as phase equilibria, enthalpy of mixing, and/or activities of solution species. Additionally, it is necessary to understand structural properties such as atomic coordination for development of a model sublattice framework. Many of these properties for the salt systems of interest are not known, however, and would be difficult and time-consuming to measure experimentally for the large range of salts under investigation, particularly when considering minor salt additives and impurities. Fortunately, molecular dynamics modelling provides a convenient and relatively easy means for the calculation of these properties.

Molecular dynamics (MD) is an atomistic modelling method in which atomic potential energies and forces between atoms are defined by an empirically fit interatomic potential (IP) and the atomic system is evolved over a period of time according to Newton's equations of motions. When sufficient data is available to construct a quality interatomic potential, IPMD calculations have been shown to perform exceptionally well in the prediction of many material properties [11-15]. Calculations quickly lose accuracy, however, when potential fitting data is scarce or non-existent. Additionally, the most commonly developed IP's are often not able to account for electronic interactions meaning IP methods may not always be suitable for the prediction of electronic properties and features that depend on electronic configuration or charge transfer such as redox states.

Ab-initio molecular dynamics (AIMD), developed in response to these issues, are essentially MD simulations where the IP is replaced with ab-initio or (first principles) DFT calculations between Newtonian timesteps. AIMD calculations have been shown to be very accurate and are also able to predict electronic properties and account for charge transfer [16,17], however, they can quickly become very computationally expensive and are usually limited to smaller system sizes and shorter simulation times.

A good example of AIMD modelling in molten salts is given by Bengtson et al. [18] who sought to evaluate the accuracy (and potential usefulness) of AIMD methods for molten salts by modelling several properties of a eutectic $\mathrm{LiCl}-\mathrm{KCl}$ salt mixture over a range of temperatures. They calculated properties such as equilibrium volume, thermal expansion, bulk modulus, self-diffusion coefficients and the Gibbs free energy of mixing. Figure 5 and Figure 6, which show the results of self-diffusion coefficient and Gibbs free energy of mixing calculations, respectively, both show close agreement with experimental data existing for the system. 
(a) $\mathrm{Li}$ in $\mathrm{LiCl}-\mathrm{KCl}$ eutectic

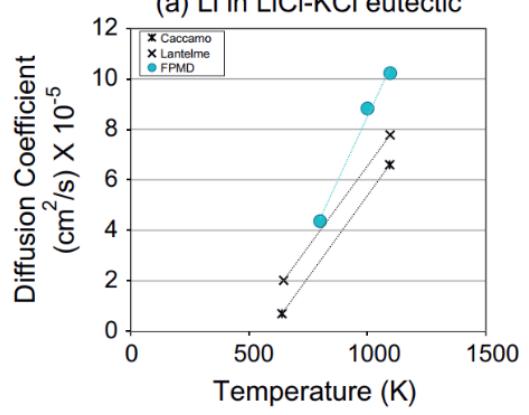

(b) $\mathrm{Cl}$ in $\mathrm{LiCl}-\mathrm{KCl}$ eutectic

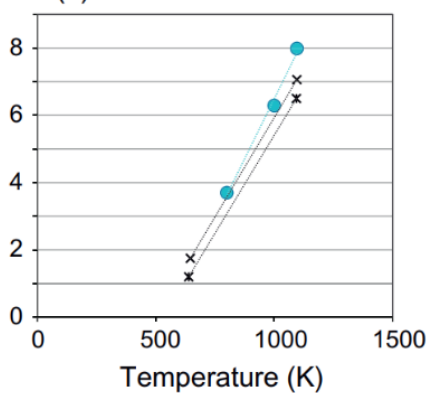

(c) $\mathrm{K}$ in $\mathrm{LiCl}-\mathrm{KCl}$ eutectic

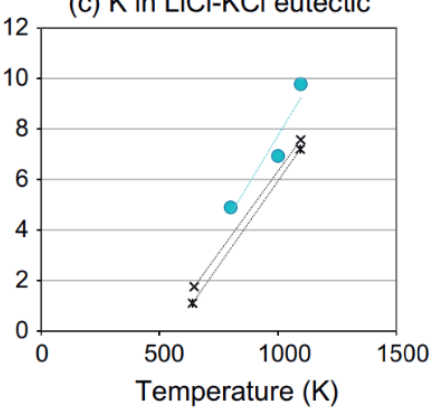

Figure 5. AIMD calculated self-diffusion coefficients for a LiCl-KCl salt mixture showing close agreement with experimental values. Figure reproduced from Bengtson et al. [18].

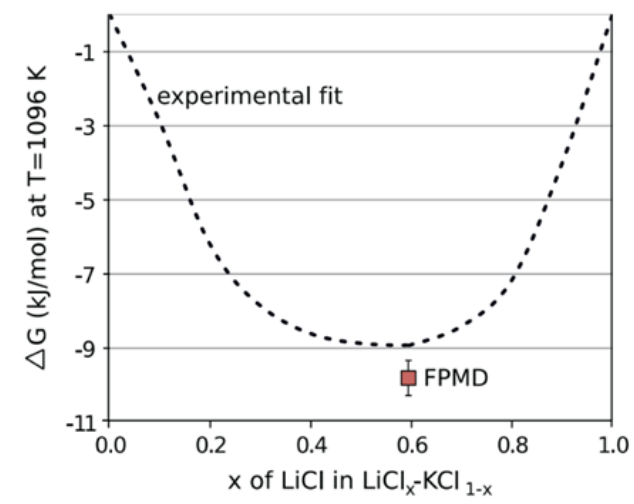

Figure 6. AIMD calculated Gibbs free energy of mixing for eutectic LiCl-KCl salt mixture at $823^{\circ} \mathrm{C}$. Figure reproduced from Bengtson et al. [18].

An interesting finding by Bengtson et al, however, who also compared their AIMD results with IPMD data, was that the AIMD methods did not significantly improve upon the results of IPMD methods when adequate experimental data was available and a well-fit IP was able to be constructed for the material system. This suggests that if sufficient data exists and if charge transfer effects can be incorporated for the current salts under investigation, it could be more efficient to spend time constructing IP's for traditional IPMD calculations than to stick to the slow and limited AIMD models.

The fitting of an accurate interatomic potential can be cumbersome and time-consuming, but the resultant IPMD simulations will be able to explore more properties using larger systems and much longer timescales than AIMD. While the necessary experimental data for many of the current salt systems of interest is limited, IP's can also be parametrized from traditional ab-initio DFT calculations. This exact approach was taken by Dewan et al. [19] who used a dipole polarization incorporating IP developed from

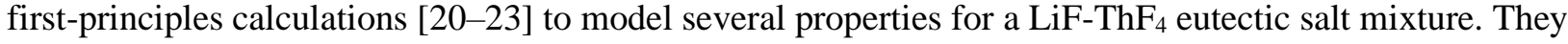
were able to accurately calculate atomic densities, thermal expansion, self-diffusion coefficients, electrical conductivity, viscosity, heat capacity and atomic coordination over a range of temperatures with simulations of over 500 atoms that ran for several thousand of picoseconds (AIMD simulations are generally limited to $\sim 200$ atoms and only a few picoseconds). 
Manga et al., [24] used traditional IPMD calculations to directly supply CALPHAD models and calculate equilibrium phase diagrams for the ternary $\mathrm{NaCl}-\mathrm{KCl}-\mathrm{ZnCl}_{2}$ system and its binary sub-systems. In this study, they calculated several of the previously mentioned properties including pair distribution functions (PDF) and coordination numbers (Figure 7) and enthalpies of mixing (Figure 8). In most salt systems, calculated PDF and coordination data like this can be directly compared to experimental XAFS (X-ray Absorption Fine Structure) data.
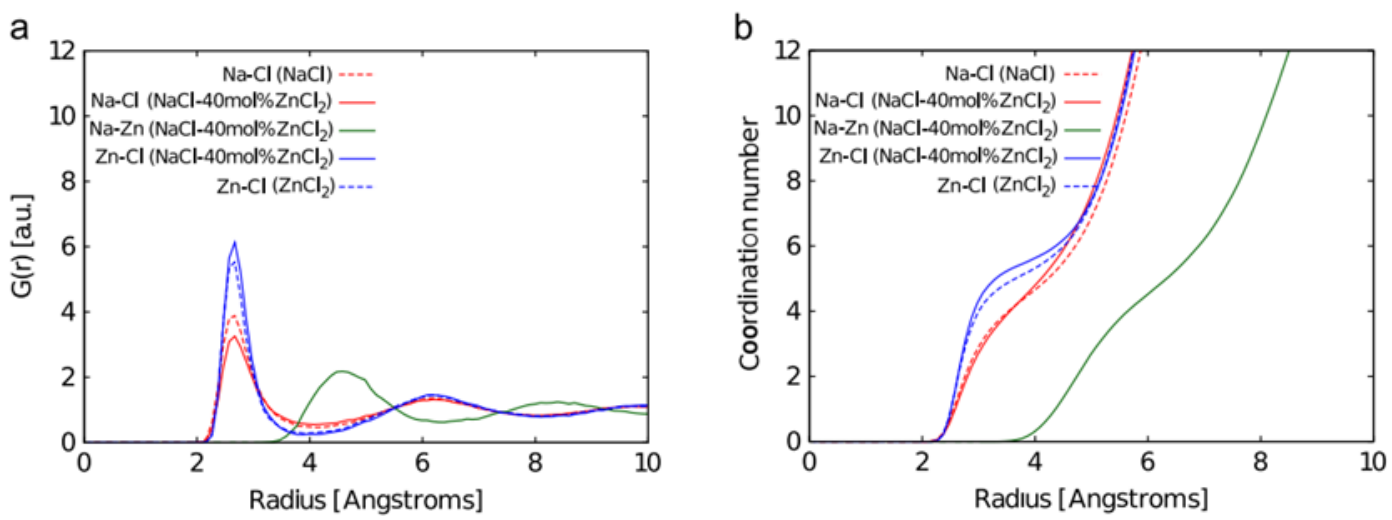

Figure 7. (a) Pair distribution functions and (b) coordination numbers calculated using IPMD methods for the $\mathbf{N a C l}-\mathbf{Z n C l}_{2}$ binary sub-system of $\mathrm{NaCl}-\mathrm{KCl}-\mathbf{Z n C l}_{2}$. Figure reproduced from Manga et al. [24].

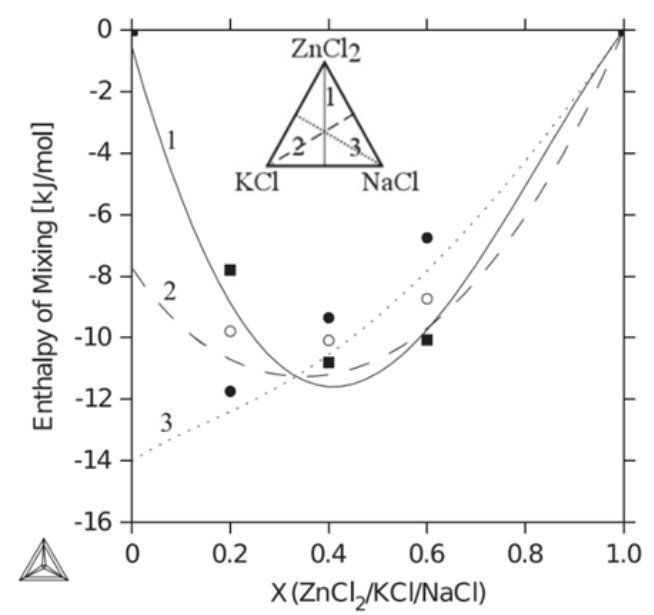

Figure 8. Enthalpies of mixing calculated using IPMD for the liquid $\mathrm{NaCl}-\mathrm{KCl}-\mathrm{ZnCl}_{2}$ ternary salt at $827^{\circ} \mathrm{C}$. The lines represent CALPHAD results and the points represent IPMD simulation results. The squares correspond to line 1, open circles to line 2, and closed circles to line 3. Figure reproduced from Manga et al. [24].

Using IPMD calculated data in a subsequent CALPHAD model, Manga et al. [24] was then able to construct the equilibrium phase diagrams for the parent ternary system and each of the binary subsystems. Figure 9 shows a calculated isothermal section of the ternary phase diagram at $250^{\circ} \mathrm{C}$ (left) and a liquidus projected ternary phase diagram showing the predicted melting temperature in Kelvin over the entire range of salt composition (right). 

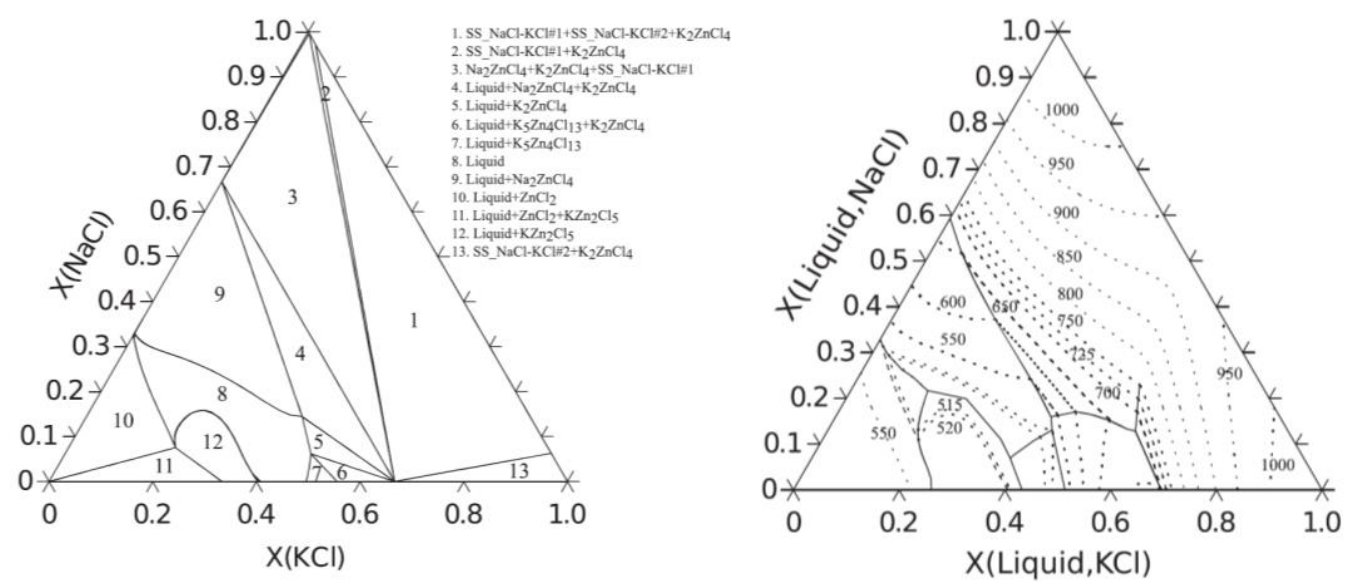

Figure 9. Equilibrium phase diagrams from IPMD and CALPHAD calculations. (left) An isothermal section of the $\mathrm{NaCl}-\mathrm{KCl}-\mathrm{ZnCl}_{2}$ ternary equilibrium phase diagram at $250^{\circ} \mathrm{C}$ and (right) a liquidus projected phase diagram for the same system showing the predicted melting temperatures (in Kelvin) from IPMD informed CALPHAD models.

Figure reproduced from Manga et al. [24].

Future salt chemistry and thermodynamic modelling efforts should focus on applying these MD methods to predicting similar properties for the leading salt mixture candidates and their binary subsystem salts. These include both non-actinide containing salt systems like $\mathrm{NaCl}-\mathrm{MgCl}_{2}, \mathrm{LiF}-\mathrm{BeF}(\mathrm{FLiBe})$ and $\mathrm{LiF}-\mathrm{NaF}-\mathrm{KF}(\mathrm{FLiNaK})$ and actinide salts like $\mathrm{UCl}_{3}-\mathrm{UCl}_{4}-\mathrm{NaCl}$ and $\mathrm{LiF}-\mathrm{BeF}_{2}-\mathrm{UF}_{4}$. Once a solid computational foundation for modelling these salt systems has been achieved, efforts need to then focus on applying the same modelling principles and techniques to calculate the effects of impurities such as corrosion or fission products on these thermophysical and thermochemical properties.

\subsection{REGION II: MASS TRANSPORT IN THE ALLOY BULK}

In the bulk region of the alloy, which can be considered the region below the outermost handful of atomic surface layers, the diffusion and migration of atoms, ions, and other defects is of principal interest. The exchange of atoms occurring at the salt interface, whether regarding alloy components leaving the alloy or salt ions penetrating into the alloy, must be facilitated by some level of diffusion through this bulk region. Examples of this are shown in Figure 3, where both $\mathrm{Cr}$ depletion depths and $\mathrm{O}$ and Na penetration depths can be seen extending several tens of micrometers into the alloy. Whether via gradient driven interstitial migration, vacancy diffusion or some other unknown processes, the underlying mechanisms driving the movement of these atoms through the bulk lattice regions are generally not well described or understood.

In the previous section, the large numbers of atoms and the necessary time lengths needed to calculate properties relevant to the salt chemistry and thermodynamics limited the choice of computational models to IPMD and AIMD methods. In the bulk region, however, many properties of interest, like migration paths and activation energies are largely dependent only on the local atomic coordination shell surrounding the migrating atom or defect where significant forces and lattice distortions might only extend up to third or fourth nearest atomic neighbors. This means much smaller 
simulation cell sizes ( $<200$ atoms) can be employed to model these properties and features allowing traditional first-principles and ab-initio methods like DFT to be used.

DFT (Density Functional Theory) is an electronic structure calculation that solves the KohnSham potential equations, an approximated form of the Schrödinger equation, for an electronic manybody system [25]. A set of atoms and atomic coordinates is given to the DFT model, and an electronic wavefunction for that configuration is calculated according to the Kohn-Sham potential equations. Forces on atoms are then calculated based on the wavefunction, and the atomic positions are updated according algorithms that seek to minimize the total energy and forces present in the system. The AIMD models discussed in the previous section make use of this by replacing the interatomic potential with an electronic wavefunction DFT calculation to determine the forces on atoms between timesteps. The difference between AIMD and traditional static DFT is in the algorithms used to update the ionic positions. AIMD employs Newton's equations of motion and allows for a true evolution of time and the movement of atoms. It does not seek a minimum energy or ground state configuration, allowing for temperature (rapid vibrations of atoms) to be accounted for. The resultant energies and properties of AIMD calculations are then averages of the final timesteps of a simulation. The algorithms used in static DFT codes, on the other hand, do not correspond to the true movement of atoms, instead seeking only a minimum energy position through whatever path it takes to get there. In this regard, time isn't really evolved in a DFT simulation and the calculation itself can be thought of as a single momentary snapshot depicting the true minimum energy ground state of the system at $0 \mathrm{~K}$. Entropy terms then vanish because of the $0 \mathrm{~K}$ temperature leaving the total energy calculated in a DFT simulation essentially equal to the internal energy of the system's ground state configuration.

DFT models have, quite successfully, been able to take advantage of this internal energy calculation to quantify and characterize atomic properties and behaviors through reference state calculations [26-29]. In these types of calculations, a total energy is calculated for some reference state configuration and used as a base to quantify the energetics of slightly altered or "perturbed" versions of that system to determine useful properties like formation energies and enthalpies, and migration paths and barriers.

Applying this framework to study molten salt induced corrosion and chemical processes in the bulk alloy could yield interesting and useful insights into the governing forces in this region. For example, the minimum energy migration paths of an atom or salt ion moving through the alloy can be directly modelled, and the effects of alloy composition or the presence of lattice defects like vacancies or interstitials on these migration paths can be quantified. This is commonly done by combining DFT total energy calculations with the Nudged Elastic Band method (NEB) [30-32]. In the NEB method, which was developed for finding saddle points and minimum energy paths through an energy surface, an approximate path is guessed and then populated with a series of points referred to as images. The images are then optimized according to an algorithm to find the lowest possible image energy. The resulting path represents the lowest possible energy path to get from an initial to final state.

An example of this can be seen in the work by Zhang et al. [27] who used DFT NEB calculations to model the formation and migration of vacancies in Re-doped $\mathrm{Ni}_{3} \mathrm{Al}$. In this study, vacancy migration paths leading to different lattice site positions in the pure $\mathrm{Ni}_{3} \mathrm{Al}$ unit cell and in two Re-doped unit cell configurations were modelled. Figure 10 shows the resultant energy paths that were calculated using the DFT NEB method. The height of the curve represents the energy barrier that must be overcome for the migration event to occur. Larger multi-scale models like $\mathrm{kMC}$ are able to make use of these kinds of relative energies in the prediction of long-term migration and diffusion properties in large atomic systems. 


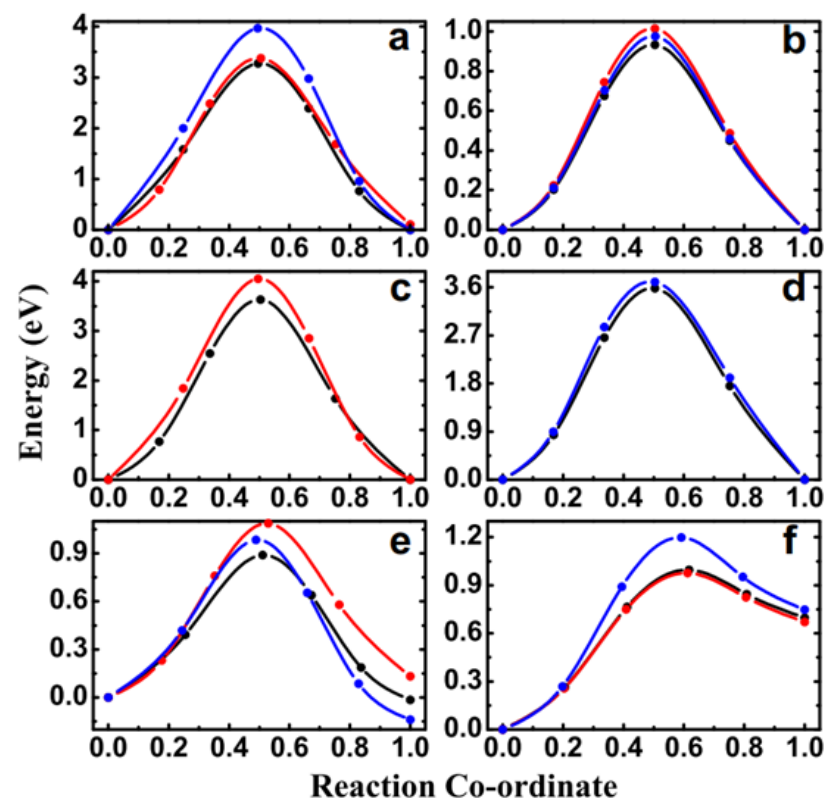

Figure 10. DFT NEB calculated vacancy migration energy paths in $\mathrm{Ni}_{3} \mathrm{Al}$. Each plot (a-f) depicts the energy path (and energy barrier) associated with the vacancy moving to a specific lattice site in the unit cell. The colors refer to the pure $\mathrm{Ni}_{3} \mathrm{Al}$ unit cell (black), a $\mathrm{Re}_{\alpha}$-doped (blue) unit cell and $\mathrm{Re}_{\beta}$-doped unit cell (red). Figure reproduced from Zhang et al. [33].

Similar to the way in which Zhang et al. incorporated the effect of Re-doping in their models, the effect of alloy concentration gradients and salt contamination can be investigated by modelling migration paths in Ni-Cr and stainless-steel alloys by use of carefully constructed simulation cells. For example, the energetics of vacancy migration in one of these alloys would be governed by the local arrangement and ordering of the first and second nearest neighbors around the vacancy site, which itself would be directly correlated to the overall alloy concentration and any concentration gradients present in that region. By using DFT NEB methods to explore migration paths in unit cells containing, say different numbers and configurations of $\mathrm{Cr}$ atoms, the direct effects of $\mathrm{Cr}$ concentration on the migration of other alloy atoms, salt constituents or even $\mathrm{Cr}$ itself can be learned. An example of this is shown in Figure 11, which depicts several simplistic unit cell configurations that could be used to glean this type of information. In each of these configurations, an energy curve can be calculated for the center $\mathrm{Cr}$ atom to migrate into the adjacent vacancy site. The height (representing the activation energy) and shape of each curve, along with the relative energies of the initial and final states will be affected by the local arrangement of neighboring atoms, represented here by the Cr cluster in Figure 11(b) and the salt atom/ion in Figure 11(c). The relative energies of these curves will suggest preferred migration mechanisms and paths and possibly provide insight into the overarching driving forces $\mathrm{Cr}$ and salt migration in the alloy. 


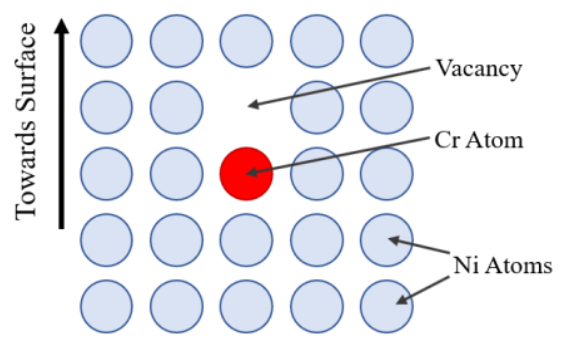

(a) Reference $\mathrm{Cr}$ migration

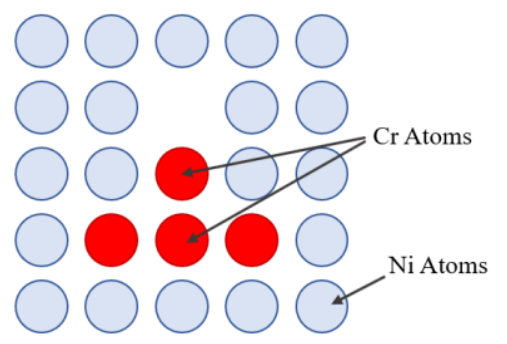

(b) Cr clustering effects

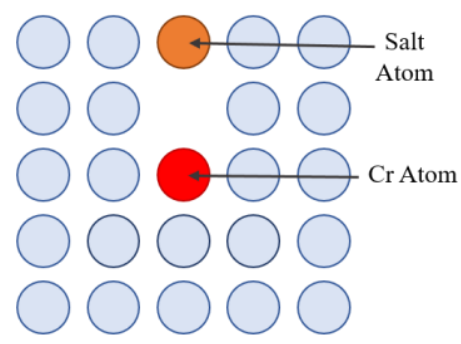

(c) Salt contaminant effects

Figure 11. Examples of atomic configurations of a $\mathrm{Ni}-\mathrm{Cr}$ alloy that could be used to model $\mathrm{Cr}$ migration energies and paths with DFT NEB methods. Configuration (a) shows what could considered a reference path or energy curve for which the other configurations can be compared to. The effects of $\mathrm{Cr}$ or other alloy constituents on Cr migration could be modelled using configurations similar to that shown in (b) and the effect of salt contaminants on the migration of atoms can be modelled with configurations similar to that seen in (c).

These types of DFT calculations can provide extremely useful and interesting data, however, it shouldn't be forgotten that they are modelled at $0 \mathrm{~K}$ temperature. While this not ideal for modelling high temperature molten salt properties, the basic and fundamental forces being calculated between atoms are still valid and can often be extrapolated to high temperature regimes using various thermodynamic principles.

While gaining a better understanding of these local forces and interactions between individual atoms would certainly be considered an accomplishment and provide useful data, they are not the only feature of bulk alloy behaviors that can be investigated with atomistic modelling. Just as AIMD and IPMD methods were suggested to model diffusion coefficients in Region I molten salts, several studies can be found demonstrating similar types of calculations for the diffusion of atoms and defects in bulk alloys [34-39]. These types simulations could provide an evaluation of diffusion coefficients at reactor temperatures and potentially offer an understanding of the relative transport mechanics of individual alloy components and salt contaminates in both the bulk region and along grain boundaries. Of course, these bulk region MD simulations will still be subject to the same constraints limiting the molten salt MD simulations in that the AIMD models will be limited to smaller system sizes and timescales and the IPMD models will only be as good as the data used to construct their interatomic potentials

\subsection{REGION III: SALT/ALLOY INTERFACE PHENOMENA}

The interface region between the liquid salt and the top two to three surface layers of the alloy is where the most fundamental and germinal corrosion driving processes must reside. While the presence of solute concentration gradients or salt contaminants in the bulk alloy and the changing chemistry and thermodynamics in the liquid salt may facilitate the corrosion process, it's the fundamental mechanisms underlying the exchange or transport of atoms across the physical interface that are the root cause of corrosion in molten salt systems. Understanding these processes and mechanisms would undoubtedly lead to a better and more efficient ability to design and test new materials for use in these systems.

As these mechanisms are likely to be characterized by the local interactions and forces between individual atoms of the surface and the ionic species and molecules of the charged liquid salt, electron 
exchange interactions and charge transfer likely play a significant role. Because of this, modelling efforts should be built around first principles models, like DFT or AIMD, that are able to model electronic behavior during these processes. These models should be focused on understanding one of three different types of environments that can exist at the interface:

(i) Just below the surface: The effects of embedded salt constituents on the electronic and physical behaviors of alloy atoms and features (e.g. defects) in the top three surface layers.

(ii) On top of the surface: The deposition and adsorption behavior of salt constituents and impurities on top of the surface.

(iii) Atomic Exchange: The direct exchange of atomic or ionic species from surface to liquid or vice-versa.

Additionally, since the specific surface environments seen by the liquid salt can vary significantly from one area to the next, each model focus should be explored over several surface environments (e.g. flat smooth surfaces, step-edges and dislocations, grain boundary terminations, etc.)

\subsubsection{Focus (i) - Just Below the Surface}

The interactions, energetics, and lattice dynamics of alloy and salt components in the top surface layers can be modelled fairly easily with traditional DFT methods. As an example, simple and easy to implement DFT calculations can be designed to investigate the segregation behavior of atoms near the surface. Atom segregation refers the propensity for atoms in the top surface layers to either move towards or away from the surface and can be quantified with surface segregation energies which describe the change in energy associated with the solute atom's position in any one of the top layers relative to a position deep in the bulk region of the material. The presence of ionic species above or adsorbed to the surface, the inclusion of salt ions in the top layers, and the arrangement of alloy atoms around the segregating atom will have an effect on this process. DFT can be used to directly calculate the changes in total system energy associated with solute layer position and surface coverage effects. Examples of these types of studies can be seen in the works of Yu et al. [40] and Guesmi et al. [41] who studied the segregation of transition metals in fcc $\mathrm{Ni}$ and the chemisorbed $\mathrm{O}$ influenced $\mathrm{Pd}$ segregation in fcc $\mathrm{PdAu}$ alloy, respectively. Figure 12 shows an example of the atomic simulation surface slab cells used by Yu et al. and Figure 13 shows the resulting segregation energies that were calculated using these slab structures. In the case of segregation energies, a negative energy means the configuration is more energetically favorable and that the segregating atom would prefer its position in the surface layer over a position in the bulk. A positive energy means the opposite and that its position in the surface layer is energetically unfavorable. 


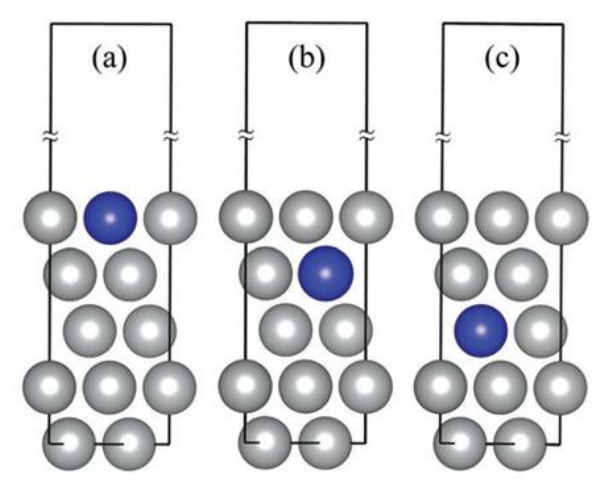

Figure 12. Surface slabs used to calculate surface segregation energies in fcc Ni. Surface slab (a) depicts the transition metal in the 1st surface layer, (b) in the 2nd layer, and (c) the 3rd layer. Figure reproduced from Yu et al. [40].
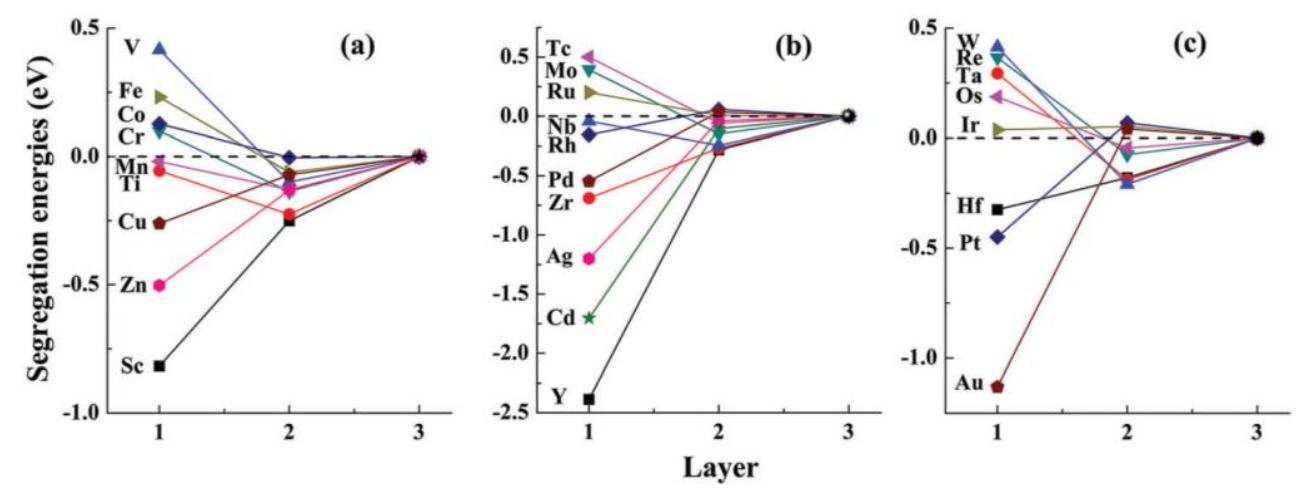

Figure 13. Calculated surface segregation energies for several transition metals in an fcc $\mathbf{N i}(111)$ surface. Plot

(a) corresponds to $3 \mathrm{~d}$ transition metals, plot (b) to $4 \mathrm{~d}$ metals and plot (c) to $5 \mathrm{~d}$ metals. In this case the transition metal positioned in the third layer is said to be equivalent to a position in the bulk region, and so the energies for positions in layers one and two were calculated in reference to the energy of the third layer configuration. Figure reproduced from Yu et al. [40].

Surface segregation calculations describe just the energetics associated with the positioning of solute atoms in and around lattice sites at the surface, but the actual migration paths and energy barriers the segregating solute atoms must traverse to get from layer to layer are also of interest and could provide useful information about lattice dynamics in the top surface layers. DFT NEB models can be used to model these energy paths in the same manor that was described for bulk migration paths in Region II. Here, the effects of the local coordination shell around the migrating atom would also be of great interest as concentration effects (alloy solute constituents and salt ion or impurity inclusions) and the presence of lattice defects like vacancies, interstitials, or dislocations could have a large effect on both the migration process and the overall energies (and lattice stability) of the initial and final states. 


\subsubsection{Focus (ii) - On Top of the Surface}

While the segregation and migration studies of focus (i) are concerned with forces and behaviors exhibited by alloy and salt components in the uppermost atomic surface layers, focus (ii) models should be concerned with atomic behaviors above and on top of the surface. These include the properties and processes associated with the adsorption and deposition of salt components on the alloy surface, which are commonly and easily modelled using first-principles methods [42-48]. While adsorption and deposition could be considered very similar processes, in the case of modelling, it is useful to classify adsorption as the adhesion of salt component ions and atoms to specific lattice sites on the top of the surface and the eventual formation of an entire surface mono-layer (ML) while deposition as the multilayer accumulation, clustering, and growth of salt constituents and impurities on the surface.

An example of an adsorption study in which the effect of surface coverage is investigated can be seen in the work of Ma et al. [48]. In this study, DFT was used to model the change in adsorption energy of $\mathrm{S}$ on a $\mathrm{Co}(0001)$ surface, and it was found that as the surface coverage (or the number of adsorbed $\mathrm{S}$ atoms) increased, the adsorption energy decreased. In the case of adsorption energies, a negative energy means it is favorable and there is an attraction between the adsorbate and surface, while a positive energy means there is repulsion between the adsorbate and surface. In the case $\mathrm{S}$ adsorbing onto $\mathrm{Co}(0001)$, the increasing surface coverage decreased the overall attraction between the surface and S adatoms. Figure 14 contains a plot of the calculated adsorption energies showing this effect.

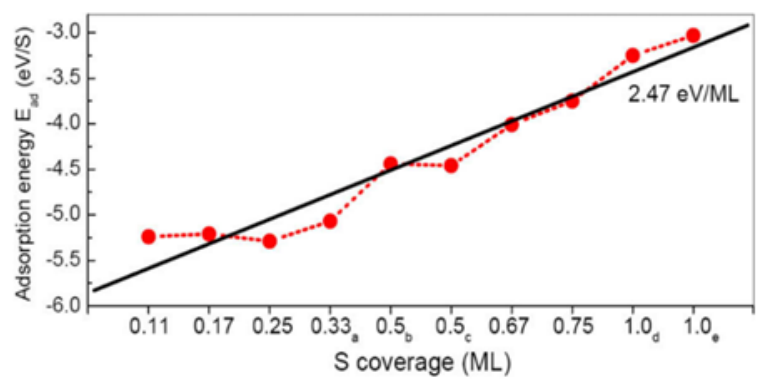

Figure 14. Calculated adsorption energy of $S$ on a Co(0001) surface from DFT. ML stands for mono-layer so that 1.0 ML corresponds a full surface mono-layer. The increasing adsorption energy here means that as the surface coverage increases the overall adsorption process becomes less energetically favorable. Figure reproduced from Ma et al. [48]

In molten salt systems, DFT adsorption studies could provide information on the propensity of different salt components and impurities to adhere to the alloy surface. Knowledge of these behaviors could then eventually be used to infer or predict the most likely species interaction candidates for corrosion processes at the surface interface.

As for deposition processes, traditional DFT models could be used to investigate the energetics associated with the size and ordering of surface clusters as they form from individual atoms and ions. However, it may be more interesting to employ an AIMD approach to study the formation and growth of these clusters so that realistic liquid salt and temperature effects can be included. Kwon et al, [42] took this very approach when they modelled nucleation and growth mechanisms of U clusters on a Mo(110) surface in contact with a liquid $\mathrm{LiCl}-\mathrm{KCl}$ molten salt. An interesting finding of this investigation, shown 
in Figure 15 and Figure 16, was that the liquid salt had a significant effect on the shape, structure, and stability of U clusters. Similar studies could be designed to extend this kind of investigation to compare the same types of effects for different salt systems (chloride versus fluoride for example) on the formation and structure of deposited $\mathrm{U}$ and (or) other impurity clusters on the surface of $\mathrm{Ni}$ and steel alloys.

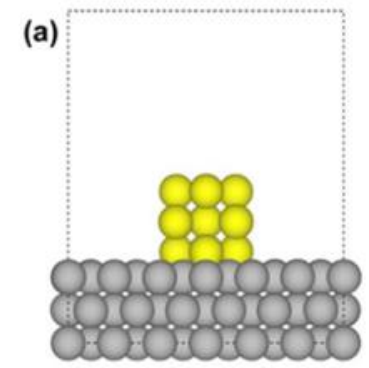

0 ps

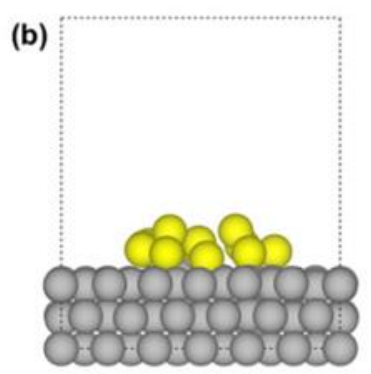

$1 \mathrm{ps}$

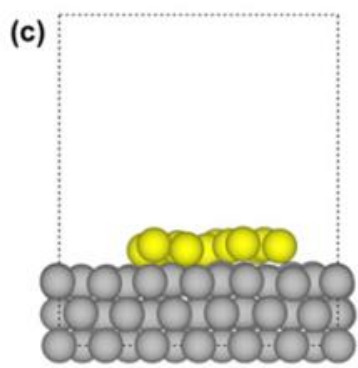

2 ps

Figure 15. AIMD simulation of $U$ nanocluster on a Mo(110) surface under a vacuum. As time evolved, the cluster flattened and lost its shape. Figure reproduced from Kwon et al. [42]

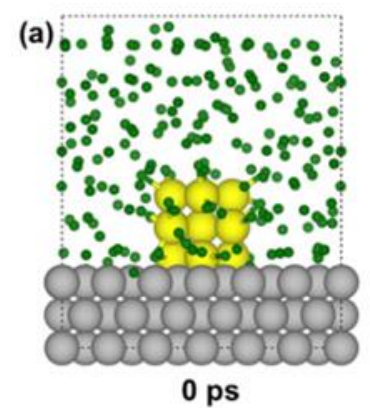

(b)

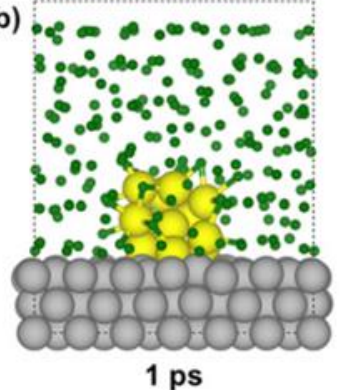

(c)

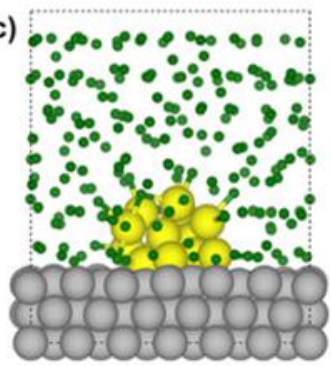

$2 \mathrm{ps}$

Figure 16. AIMD simulation of $\mathrm{U}$ nanocluster on a Mo(110) surface under a LiCl-KCl liquid salt. As time evolved, the initially rigid and box shaped cluster compacted slightly into the shape of a sphere. The important observation, however, is that due to the presence of the liquid salt, the U maintained its overall "cluster" shape and structure. Figure reproduced from Kwon et al. [42]

Similar AIMD approaches could also be taken to model salt adsorption characteristics for different surface structures (flat surfaces, step-edges, etc.) focusing specifically on the competition between different salt species for preferred adsorption sites and overall coverage.

\subsubsection{Focus (iii) - Atomic Exchange}

Focus (iii) models, concerned with the specific exchange of atoms, ions, and electrons at the surface interface, could provide arguably the most exciting and potentially interesting information about the underlying corrosion mechanisms in molten salts. While these exact mechanisms may not be known, leading to some potential uncertainty in the results and conclusions of these models, by modelling 
relatively simple reaction pathways that are known to occur for other material systems, and directly comparing the different effects and properties observed for each type of salt and alloy components within these pathways, valuable insights into which of these component species are the most fundamentally linked to the corrosion process could be obtained.

Models could be designed, for example, to compare the dynamics of salt component trapping in surface vacancies, along edge dislocations protruding from the surface, or within surface cracks. The configuration of alloy components around these vacancy or edge locations could then be changed to test the salt entrapment properties of the various metals that make up the alloy surface (e.g. $\mathrm{Cr}, \mathrm{Mo}, \mathrm{Ni}, \mathrm{Fe}$, etc...). Extending this model, the subsequent migration of adsorbed or entrapped salt ions (or the migration of alloy atoms to the salt ion) across edge steps or through vacancy or dislocations loops could then be modelled. Lastly, as a final step in this series, the dissolution of the salt ion with an alloy atom could be investigated. A similar study was carried out by Pavlova et al. [47] who used traditional DFT to model, first, the dissociation and adsorption of $\mathrm{Cl}_{2}$ onto a $\mathrm{Cu}(111)$ surface, and then the subsequent dissolution of $\mathrm{Cl}, \mathrm{Cu}, \mathrm{Cl}_{2}, \mathrm{CuCl}$ and $\mathrm{CuCl}_{2}$ species from the surface. The calculations were carried out over various flat surface and step-edge dislocation configurations finding that, of the dissolution products, $\mathrm{CuCl}$ faced the lowest dissolution energy barrier and that overall dissolution preferentially occurs at step edges and places where the dissolving $\mathrm{Cu}$ atom has a reduced coordination number relative to the perfectly flat surface. Figure 17 shows an example of the dissolution energies calculated for each type of dissolution product as a function of total $\mathrm{Cl}$ surface coverage.

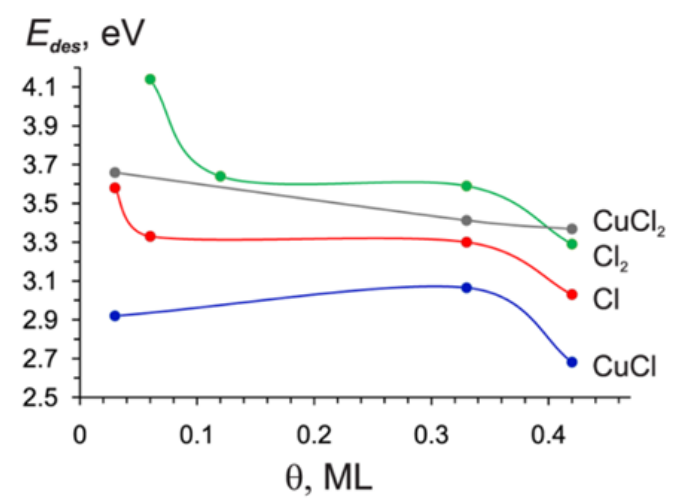

Figure 17. DFT calculated dissolution (desorption) energies for several dissolution products for a $\mathrm{Cu}(111)$ as function of $\mathbf{C l}$ surface coverage $(\boldsymbol{\theta})$. The energies depicted here $\left(E_{d e s}\right)$ are equivalent to the height of the energy barriers that each product must overcome in order to desorb. The $\mathrm{CuCl}$ molecule therefore has the highest likelihood of dissolving, relative to the other modeled products, for the entire range of $\mathrm{Cl}$ surface coverage. Figure reproduced from Pavlova et al. [47]

In addition to surface dissolution energies, the work of Sangiovanni et al. [49], which demonstrates the application of AIMD in the identification and comparison of surface reaction pathways that facilitate metal organic chemical vapor deposition (MOCVD) processes, represents another potential modelling framework that could be employed to explore molten salt corrosion mechanisms. In this work, an AIMD model was used to identify and characterize reaction pathways for the surface epitaxy of AlN on a graphene sheet at elevated temperatures. The authors modelled several possible surface reaction pathways involving various molecular species associated with the process and characterized the electron 
charge transfer during each reaction, one of which, depicting the adsorption of $\mathrm{CH}_{3}-\mathrm{Al}-\mathrm{CH}_{2}$ on graphene, is shown in Figure 18.
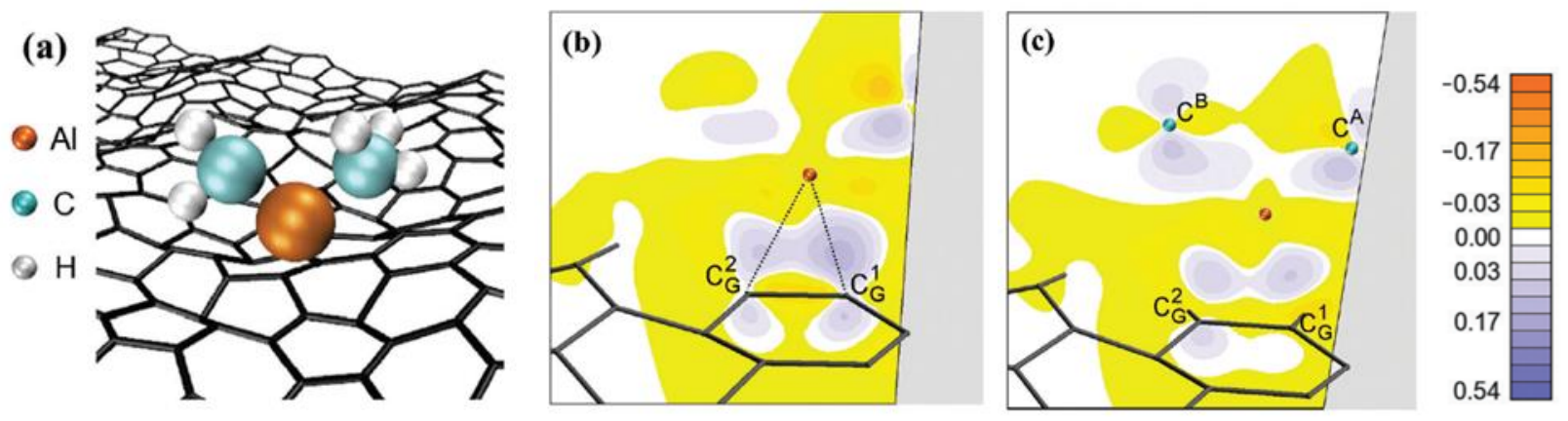

Figure 18. AIMD simulation of the adsorption of a $\mathrm{CH}_{3}-\mathrm{Al}-\mathrm{CH}_{2}$ molecule on graphene. Image (a) depicts a snapshot image of the atoms and structure of the $\mathrm{CH}_{3}-\mathrm{Al}-\mathrm{CH}_{2}$ and graphene sheet (black bonds). Images (b) and (c) shows the evolution of electron charge transfer between the molecule and graphene sheet at two different points in the reaction pathway. The images are several femtoseconds apart. The electron density color scheme, expressed in units of $\left[\mathrm{e}^{-} \AA^{-3}\right]$, shows negative values with red/yellow and positive values with grey/blue. $C_{G}^{1,2}$ label the graphene carbon atoms and $C^{A, B}$ the gas phase carbon atoms. Figure reproduced from Sangiovanni et al. [49].

Similar AIMD investigations could be designed to investigate potential corrosion reaction pathways in molten salts. Processes related the adsorption, diffusion, and penetration of salt ions and the dissolution of salt and alloy complexes could be modelled in this manner. The role and importance of charge transfer in these salt/alloy surface interactions could be directly evaluated in these approaches for several different types of salt ion species and alloy component atoms. While the prospect of discovering the actual corrosion mechanisms may be somewhat unrealistic, or at least unlikely to receive experimental verification, the important atom-to-atom and atom-to-ion forces between the participating species could still be analyzed for fundamental trends and behaviors that are likely to govern any type of reaction occurring at the interface.

\subsection{CONNECTING REGIONS WITH NON-EQUILIBRIUM MODELLING}

While the models discussed for Regions I-III offer the ability to calculate independent atomic driving forces and localized properties, the overall corrosion process and chemical state of a molten salt system is undoubtedly of a non-equilibrium nature. The purpose of dividing the MSR environment into three regions was to essentially allow for the separation of contributing variables along the corrosion chain making it possible to employ simple single-process or property focused computational models to investigate fundamental driving forces. Any attempt to model long-term corrosion processes or molten salt chemistry, however, would require a re-coupling of these region variables to account for the dependencies spanning region borders. For example, the overall long-term dissolution behavior of $\mathrm{Cr}$ atoms entering the salt would depend on the collective outcomes of all the individual processes occurring within each region involving the movement of $\mathrm{Cr}$ atoms. The number of $\mathrm{Cr}$ atoms in the salt would be dependent on both the overall solubility and speciation behavior of $\mathrm{Cr}$ in the salt and on the availability of $\mathrm{Cr}$ atoms at the surface of the alloy available for dissolution, which itself would be dependent on the diffusivity behavior of $\mathrm{Cr}$ through the bulk and near surface regions of the alloy. This would also depend 
on the actual $\mathrm{Cr}$ dissolution mechanism as well, which could be dependent on many other variables related to the chemical or structural environment of both the salt and alloy.

Modelling these interconnected processes can be difficult and often requires a complete and indepth understanding of atomic behaviors. Additionally, the timescales able to be modelled with ab-initio and MD simulations are not long enough to model the long-term cumulative behaviors of these processes. In order to simulate long timescales, multi-scale models, which incorporate the results of the physicsbased atomistic models used in Regions I-III into larger models, such as kinetic Monte Carlo (kMC) or finite-element methods can be used [50]. KMC, a rate-driven model, is more than capable of simulating the timescales required for many of the atomic processes associated with molten salt corrosion, such as $\mathrm{Cr}$ depletion and dissolution behaviors at the surface (Figure 19).

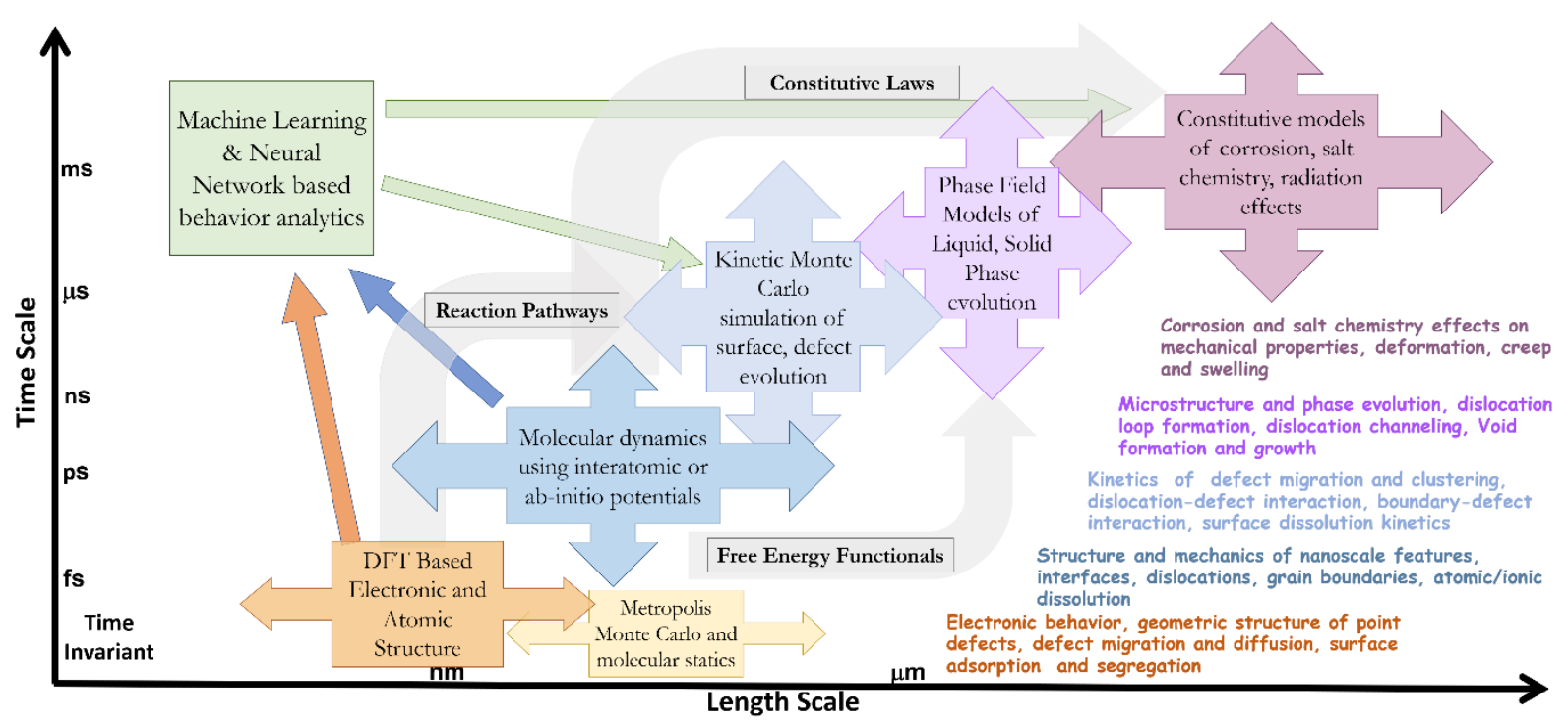

Figure 19. Relative length- and timescales of several of the most commonly employed computational materials science models that can be incorporated into multi-scale modelling. Each box represents a different modelling method and the length of the arrows extending form each box depict the respective length and time scales that each model is able to cover. The arrows travelling from one box to another box (or to a general area) show how the lower models on the ladder can be used to inform the higher and larger scale models.

The difficulty associated with $\mathrm{kMC}$, however, is that a very accurate and thorough understanding of all the potential atomic behaviors occurring in a system must be known. These models are driven by rate-related properties like energy barriers and relative energy states corresponding to the configuration and movement of atoms. This difficulty is compounded for systems containing many different types of atoms and elements over high temperature ranges, like molten salt systems, for which there can often be several hundred potential nearest neighbor configurations around a single migrating atom. Each of these configurations could present a different energy barrier to the migration of that atom for any of its potential migration coordinate pathways. An accurate molten salt corrosion $\mathrm{kMC}$ model, would require a very large database of this kind of atomic level behavior over all the physical environments in the systems (liquid salt, alloy, interface, etc.). 
While this description makes the task of developing a molten salt kMC model seem daunting or even impossible, there are features of these systems for which adequately informed kMC models could potentially be developed. For instance, a thorough ab-initio evaluation of dissolution behavior at the interface, like that discussed in the Focus (iii) section of Region III, could be used to inform a kMC model to investigate the intermediate-timescale dissolution of alloy surface features, like step-edges and grain boundary terminations. Chen et al. [51] used this exact approach to model the dissolution of $\mathrm{NaCl}$ in water. In this work, they used an AIMD model to first calculate the dissolution energy barrier for several surface structure configurations involving step-edges, vacancies, and kink sites (Figure 20 and Figure 21).

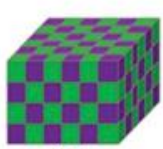

(a)

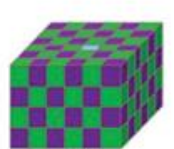

(b)

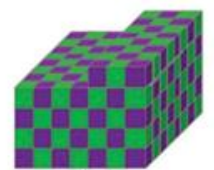

(c)

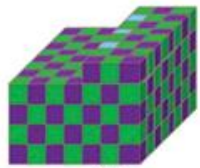

(d)

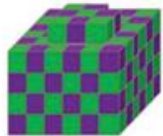

(e)

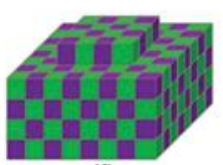

(f)

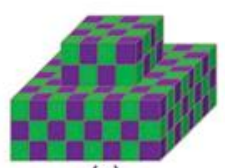

(g)

Figure 20. Different $\mathrm{NaCl}(100)$ surface structures used to model dissolution. Each structure presents a new dissolution site with a unique coordination shell. Figure reproduced from Chen et al. [51]

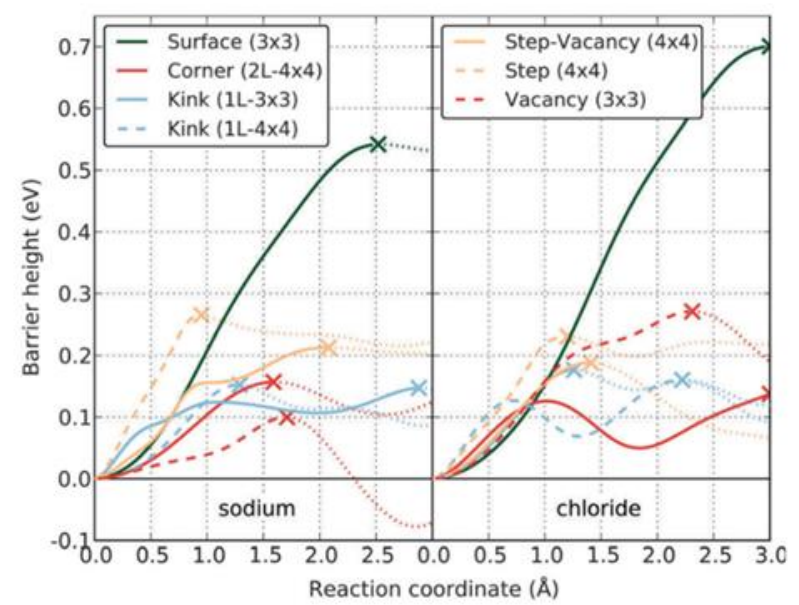

(a)

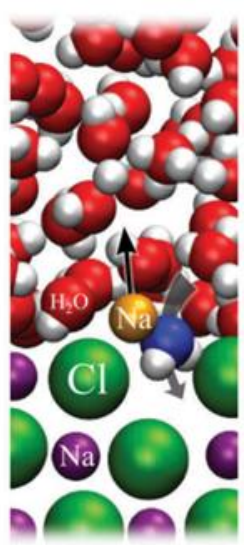

(b)

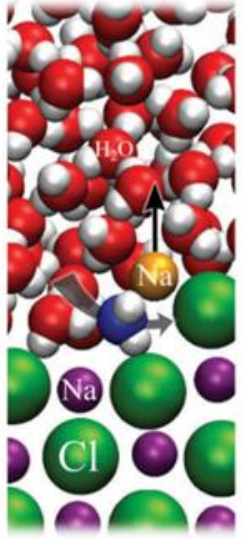

(c)

Figure 21. Results of AIMD modelling of $\mathrm{NaCl}$ dissolution in Water. (a) relative energy barriers for the dissolution of a $\mathrm{Na}$ atom (left) or $\mathrm{Cl}$ atom (right) from the various surface sites studied by Chen et al.. (b) and (c) show snapshots of the dissolution process during the AIMD simulation. Figure reproduced from Chen et al. [51]

The newly acquired dissolution energy barrier information was then used to inform a kMC model for simulating the dissolution of an $\mathrm{NaCl}$ nanocrystal over serval hundred pico-seconds (Figure 22). 


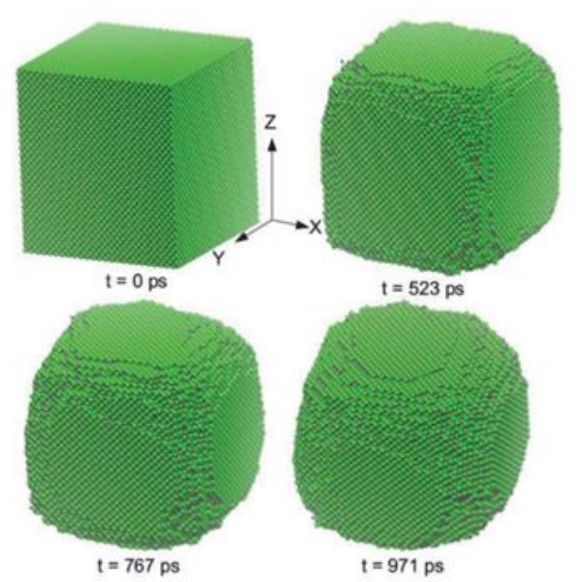

(a) KMC snapshots

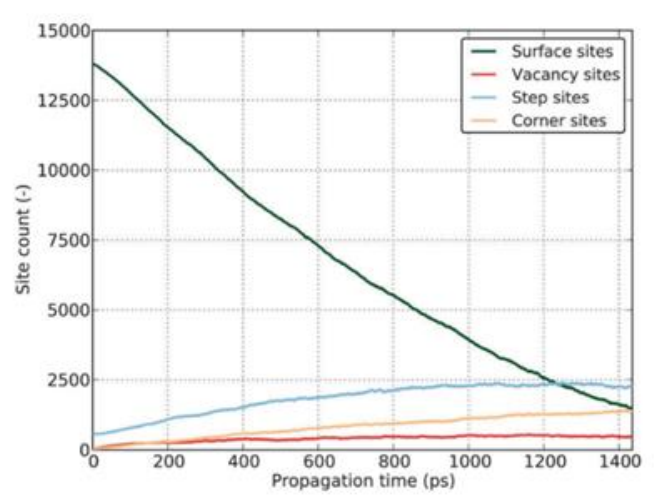

(b) Evolution of site types

Figure 22. kMC modelled dissolution of an $\mathrm{NaCl}$ nanocrystal in water. (a) depicts the degradation of the crystal surface at different times during the simulation and (b) depicts the evolution of surface sites (flat surface, vacancy hole, steps, kinks etc.) during the dissolution. Figure reproduced from Chen et al. [51].

Similar multi-scale models could be developed to study the dissolution kinetics of molten salt interfaces. Of course, an important characteristic of these systems which would need to be included, but which was not necessary to study $\mathrm{NaCl}$ dissolution, is the surface alloy solute concentration and the potential effect of solute migration, such as that of the $\mathrm{Cr}$ behavior observed in molten salt corroded $\mathrm{Ni}-\mathrm{Cr}$ alloys. These features could potentially be incorporated into kMC models if large enough and accurate enough migration path and segregation information databases can be built from ab-initio models or experimental studies. A problem with this, however, is that the sheer number of potential atomic environments and migration pathways for which energy barrier information would need to be known, could make this Modelling process drastically inefficient and time-consuming or simply unable to be done.

In some cases, though, it may be possible to significantly reduce the number of ab-initio simulations and overall computational cost through the use of machine learning or neural network methods, such as in the work of Boes et al. [52] who modelled surface segregation in AuPd (111) surfaces with a kMC model informed by a neural network trained on DFT simulations. In this work, DFT was used to calculate total energies for several different configurations of atomic ordering in the AuPd surface over the entire alloy concentration range. They determined that for the surface cell they were using, there were over 62,706 energy and lattice unique configurations, far too many to model with DFT. They were able to reduce this number, however, by hand selecting 360 specific configurations for which they calculated DFT total energies and using them to train a neural network (NN) to predict the total energy of the remaining 62,346 configurations. After optimizing their NN, they were then able to model the segregation behavior in AuPd surfaces using a kMC simulation informed by the NN. The results obtained from their final kMC model agree very well with experiment, as shown in Figure 23, which shows the $\mathrm{Au}$ fraction surface profile as a function of overall Au bulk concentration. 


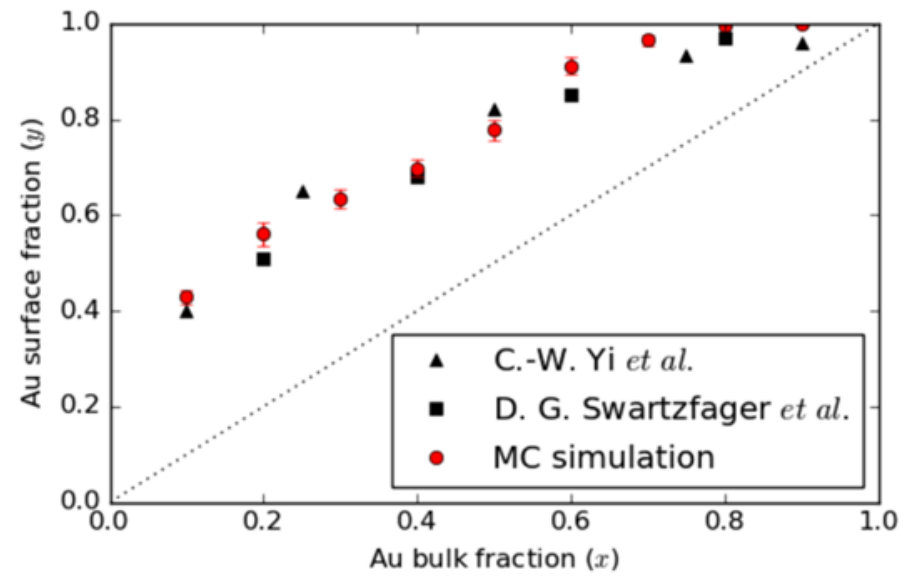

Figure 23. Segregation profile of Au atoms in a AuPd (111) surface at $527^{\circ} \mathrm{C}$. (Red Circles) are NN informed kMC simulation, (black circles squares) are experimental measurements. Figure reproduced from Boes et al. [52]

It could be possible to apply a similar approach to model the depletion behavior of $\mathrm{Cr}$ atoms in MSR systems. Whether Cr migration and depletion is driven by concentration gradients or sub-surface salt interactions, the physics-based ab-initio models discussed in the earlier sections of this report would contain this kind of information and could be used to train a neural network that could be implemented in a kMC model to evaluate the intermediate to long timescales of the depletion process. This type of NN informed segregation/depletion kMC model could also potentially be combined with the previously discussed surface dissolution type kMC model to form a fully encompassing molten salt corrosion kinetics model. It's important not to forget, though, that a kMC model of this nature can only be as accurate as the energy and rate-related data used to inform it, so before any attempt can be made to model corrosion in molten salt systems using these methods, a significant amount of time and effort must first be dedicated to developing and evaluating the accuracy of the ab-initio calculations that would be used to inform these kMC models.

\section{CONCLUSIONS}

The revitalized interest in molten salt technology over the last decade has resulted in a scientific effort across the US DOE national laboratory and US university systems to find answers to the remaining materials-based engineering questions to enable industrial deployment. At the forefront of these questions are concerns about the long-term chemical and thermodynamic stability of the salt and the degradative corrosive interactions of the salts with container materials. Experimental efforts to investigate many of these material systems are already underway, however, the high-temperatures and volatile environments inherent to molten salt systems can result in these experimental efforts being challenging, timeconsuming, and selective.

Thermodynamic modelling of salt systems would provide valuable insights into salt stability and long-term tractability. The calculation of phase diagrams and solubility behavior could then provide clues about container surface reactants and products, which could help to explore corrosion mechanism pathways. Led by these thermodynamic insights and new experimental observations, ab-initio based modelling of surface and sub-surface interactions and behaviors could be employed to investigate the fundamental driving forces behind the degradation and corrosion of container materials and the subsequent changes in salt chemistry. Multi-scale modelling techniques, informed by new computational 
data, could then be employed to investigate how slight changes in salt chemistry, salt purity, and alloy composition affect the overall degradation and long-term stability of components in these material systems.

Due to the already extensive collection of studies employing computational modelling for material systems relevant to or similar to molten salt systems, very little time would need to be spent on developing new modelling techniques or frameworks. Since experimental data for these systems is also relatively limited initial modelling investigations should be restricted to physics-based ab-initio calculations that would not require experimental or empirical parameter fitting. Additionally, since electron charge transfer is likely to play a significant role in corrosion and salt chemistry processes, electronic structure models like DFT should be employed over interatomic potential based models when possible. Lastly, once a thorough and complete understanding of the localized atomic forces driving system behaviors has been quantitively obtained, larger length- and timescale models, such as kMC, can be employed to model non-equilibrium processes and behaviors in these systems.

A computational effort employing the modelling techniques discussed in this report can be a great complement to experimental research on salt-material interactions, aiding and accelerating the development of molten salt reactor technology by enabling better and faster lifetime predictions, informing reactor safety models, and accelerating the development and testing of new materials and reactor chemistries.

\section{ACKNOWLEDGEMENTS}

The authors would like to thank Valentino Cooper for his guidance and advice. Vyacheslav Bryantsev and Stephen Utlak provided useful comments on the manuscript. This work was supported by the U.S. Department of Energy, Office of Nuclear Energy, Molten Salt Reactor Campaign.

\section{REFERENCES}

[1] The Generation IV International Forum. https://www.gen-4.org/gif. Accessed 29 May 2019.

[2] R.O. Scarlat, P.F. Peterson, The current status of fluoride salt cooled high temperature reactor (FHR) technology and its overlap with HIF target chamber concepts, Nucl. Instruments Methods Phys. Res. Sect. A Accel. Spectrometers, Detect. Assoc. Equip. 733 (2014) 57-64. doi:10.1016/j.nima.2013.05.094.

[3] D. Williams, P.F. Britt, Molten Salt Chemistry Workshop, Oak Ridge, TN, 2017. cite: https://www.ornl.gov/sites/default/files/Molten Salt Workshop_Final_092917_prelim with App A_1.pdf.

[4] E.S. Bettis, W.B. Cottrell, E.R. Mann, J.L. Meem, G.D. Whitman, The Aircraft Reactor Experiment-Operation, Nucl. Sci. Eng. 2 (1957) 841-853. doi:10.13182/nse57-a35497.

[5] P.N. Haubenreich, J.R. Engel, Experience with the Molten-Salt Reactor Experiment, Nucl. Appl. Technol. 8 (1970) 118-136. doi:10.13182/nt8-2-118.

[6] B.A. Pint, J.W. McMurray, A.W. Willoughby, J.M. Kurley, S.R. Pearson, M.J. Lance, D.N. Leonard, H.M. Meyer, J. Jun, S.S. Raiman, R.T. Mayes, Re-establishing the paradigm for 
evaluating halide salt compatibility to study commercial chloride salts at $600^{\circ} \mathrm{C}-800^{\circ} \mathrm{C}$, Mater. Corros. (2019) 1-11. doi:10.1002/maco.201810638.

[7] G. Zheng, B. Kelleher, G. Cao, M. Anderson, T. Allen, K. Sridharan, Corrosion of 316 stainless steel in high temperature molten Li 2 BeF 4 (FLiBe) salt, J. Nucl. Mater. 461 (2015) 143-150. doi:10.1016/j.jnucmat.2015.03.004.

[8] A.D. Pelton, S.A. Degterov, G. Eriksson, C. Robelin, Y. Dessureault, The modified quasichemical model I—Binary solutions, Metall. Mater. Trans. B. 31B (2000) 651-659. doi:10.1007/s11663000-0103-2.

[9] A.D. Pelton, P. Chartrand, The modified quasi-chemical model: Part II: Multicomponent solutions, Metall. Mater. Trans. A. 32 (2001) 1355-1360. doi: 10.1007/s11661-001-0226-3.

[10] C.W. Bale, E. Bélisle, P. Chartrand, S.A. Decterov, G. Eriksson, A.E. Gheribi, K. Hack, I.H. Jung, Y.B. Kang, J. Melançon, A.D. Pelton, S. Petersen, C. Robelin, J. Sangster, P. Spencer, M.A. Van Ende, Reprint of: FactSage thermochemical software and databases, 2010-2016, Calphad Comput. Coupling Phase Diagrams Thermochem. 55 (2016) 1-19. doi:10.1016/j.calphad.2016.07.004.

[11] V. Cristiglio, L. Hennet, G.J. Cuello, I. Pozdnyakova, M.R. Johnson, H.E. Fischer, D. Zanghi, D.L. Price, Local structure of liquid CaAl2O4 from ab initio molecular dynamics simulations, J. Non. Cryst. Solids. 354 (2008) 5337-5339. doi:10.1016/j.jnoncrysol.2008.04.054.

[12] N. Sun, L. Stixrude, N. de Koker, B.B. Karki, First principles molecular dynamics simulations of diopside (CaMgSi2O6) liquid to high pressure, Geochim. Cosmochim. Acta. 75 (2011) 37923802. doi:10.1016/j.gca.2011.04.004.

[13] B.B. Karki, L.P. Stixrude, Viscosity of MgSiO3 liquid at earth's mantle conditions: Implications for an early magma ocean, Science (80-. ). 328 (2010) 740-742. doi:10.1126/science.1188327.

[14] A. Klix, A. Suzuki, T. Terai, Study of tritium migration in liquid Li2BeF4 with ab initio molecular dynamics, Fusion Eng. Des. 81 A (2006) 713-717. doi:10.1016/j.fusengdes.2005.09.034.

[15] A.P. Moore, C. Deo, M.I. Baskes, M.A. Okuniewski, D.L. McDowell, Understanding the uncertainty of interatomic potentials' parameters and formalism, Comput. Mater. Sci. 126 (2017) 308-320. doi:10.1016/j.commatsci.2016.09.041.

[16] J. Qin, S. Pan, Y. Qi, T. Gu, The structure and thermodynamic properties of liquid Al-Si alloys by ab initio molecular dynamics simulation, J. Non. Cryst. Solids. 433 (2016) 31-37. doi:10.1016/j.jnoncrysol.2015.11.032.

[17] X. Lv, Z. Han, J. Chen, L. Jiang, Z. Xu, Q. Liu, First-principles molecular dynamics study of ionic structure and transport properties of LiF-NaF-AlF 3 molten salt, Chem. Phys. Lett. 706 (2018) 237-242. doi:10.1016/j.cplett.2018.06.005.

[18] A. Bengtson, H.O. Nam, S. Saha, R. Sakidja, D. Morgan, First-principles molecular dynamics Modelling of the LiCl-KCl molten salt system, Comput. Mater. Sci. 83 (2014) 362-370. doi:10.1016/j.commatsci.2013.10.043.

[19] L.C. Dewan, C. Simon, P.A. Madden, L.W. Hobbs, M. Salanne, Molecular dynamics simulation of the thermodynamic and transport properties of the molten salt fast reactor fuel LiF-ThF4, J. 
Nucl. Mater. 434 (2013) 322-327. doi:10.1016/j.jnucmat.2012.12.006.

[20] M. Salanne, C. Simon, P. Turq, R.J. Heaton, P.A. Madden, A first-principles description of liquid BeF2 and its mixtures with LiF: 2. Network formation in LiF-BeF2, J. Phys. Chem. B. 110 (2006) 11461-11467. doi:10.1021/jp061002u.

[21] V. Sarou-Kanian, A.L. Rollet, M. Salanne, C. Simon, C. Bessada, P.A. Madden, Diffusion coefficients and local structure in basic molten fluorides: in situ NMR measurements and molecular dynamics simulations, Phys. Chem. Chem. Phys. 11 (2009) 11501-11506. doi:10.1039/b912532a.

[22] M. Salanne, C. Simon, P. Turq, P.A. Madden, Heat-transport properties of molten fluorides: Determination from first-principles, J. Fluor. Chem. 130 (2009) 38-44. doi:10.1016/j.jfluchem.2008.07.013.

[23] M. Salanne, C. Simon, H. Groult, F. Lantelme, T. Goto, A. Barhoun, Transport in molten LiFNaF-ZrF4 mixtures: A combined computational and experimental approach, J. Fluor. Chem. 130 (2009) 61-66. doi:10.1016/j.jfluchem.2008.07.005.

[24] V.R. Manga, S. Bringuier, J. Paul, S. Jayaraman, P. Lucas, P. Deymier, K. Muralidharan, Molecular dynamics simulations and thermodynamic Modelling of $\mathrm{NaCl}-\mathrm{KCl}-\mathrm{ZnCl} 2$ ternary system, Calphad Comput. Coupling Phase Diagrams Thermochem. 46 (2014) 176-183. doi:10.1016/j.calphad.2014.04.004.

[25] W. Kohn, L.J. Sham, Self-Consistent Equations Including Exchange and Correlation Effects*, Phys. Rev. 140 (1965) A1133-A1138.

[26] K.S. Yoon, Y.K. Kim, T. Won, Ab-initio study on indium diffusion in silicon substrate under hydrostatic stress, Mol. Simul. 34 (2008) 47-50. doi:10.1080/08927020801895377.

[27] X. Zhang, C.Y. Wang, First-principles study of vacancy formation and migration in clean and Redoped $\gamma^{\prime}$-Ni3Al, Acta Mater. 57 (2009) 224-231. doi:10.1016/j.actamat.2008.08.052.

[28] X.X. Yu, C.Y. Wang, The effect of alloying elements on the dislocation climbing velocity in Ni: A first-principles study, Acta Mater. 57 (2009) 5914-5920. doi:10.1016/j.actamat.2009.08.019.

[29] H.M. Le, N.H. Vu, B.T. Phan, Migrations of oxygen vacancy in tungsten oxide (WO3): A density functional theory study, Comput. Mater. Sci. 90 (2014) 171-176.

doi:10.1016/j.commatsci.2014.04.018.

[30] H. Jónsson, G. Mills, K.W. Jacobsen, Nudged Elastic Band Method for Finding Minimum Energy Paths of Transitions, in: B.J. Berne, G. Ciccotti, D.F. Coker (Eds.), Class. Quantum Dyn. Condens. Phase Simulations, World Scientific, 1998.

[31] G. Henkelman, B.P. Uberuaga, H. Jónsson, Climbing image nudged elastic band method for finding saddle points and minimum energy paths, J. Chem. Phys. 113 (2000) 9901-9904. doi:10.1063/1.1329672.

[32] D. Sheppard, R. Terrell, G. Henkelman, Optimization methods for finding minimum energy paths, J. Chem. Phys. 128 (2008) 1-10. doi:10.1063/1.2841941. 
[33] S. Lu, H. Zhang, Q.M. Hu, M.P.J. Punkkinen, B. Johansson, L. Vitos, Magnetic effect on the interfacial energy of the $\mathrm{Ni}\left(\begin{array}{lll}1 & 1 & 1\end{array}\right) / \mathrm{Cr}\left(\begin{array}{lll}1 & 1 & 0\end{array}\right)$ interface, J. Phys. Condens. Matter. 26 (2014). doi:10.1088/0953-8984/26/35/355001.

[34] L. Barnard, D. Morgan, Ab initio molecular dynamics simulation of interstitial diffusion in Ni-Cr alloys and implications for radiation induced segregation, J. Nucl. Mater. 449 (2014) 225-233. doi:10.1016/j.jnucmat.2013.10.022.

[35] S. Zhao, Y. Osetsky, Y. Zhang, Preferential diffusion in concentrated solid solution alloys: NiFe, NiCo and NiCoCr, Acta Mater. 128 (2017) 391-399. doi:10.1016/j.actamat.2017.01.056.

[36] S. Zhao, G. Velisa, H. Xue, H. Bei, W.J. Weber, Y. Zhang, Suppression of vacancy cluster growth in concentrated solid solution alloys, Acta Mater. 125 (2017) 231-237. doi:10.1016/j.actamat.2016.11.050.

[37] S. Zhao, Y. Zhang, W.J. Weber, Stability of vacancy-type defect clusters in Ni based on firstprinciples and molecular dynamics simulations, Scr. Mater. 145 (2018) 71-75. doi:10.1016/j.scriptamat.2017.10.003.

[38] Y.N. Osetsky, L.K. Béland, R.E. Stoller, Specific features of defect and mass transport in concentrated fcc alloys, Acta Mater. 115 (2016) 364-371. doi:10.1016/j.actamat.2016.06.018.

[39] D.S. Aidhy, C. Lu, K. Jin, H. Bei, Y. Zhang, L. Wang, W.J. Weber, Point defect evolution in Ni, $\mathrm{NiFe}$ and NiCr alloys from atomistic simulations and irradiation experiments, Acta Mater. 99 (2015) 69-76. doi:10.1016/j.actamat.2015.08.007.

[40] Y. Yu, W. Xiao, J. Wang, L. Wang, Understanding the surface segregation behavior of transition metals on Ni(111): A first-principles study, Phys. Chem. Chem. Phys. 18 (2016) 26616-26622. doi:10.1039/c6cp02983c.

[41] H. Guesmi, C. Louis, L. Delannoy, Chemisorbed atomic oxygen inducing Pd segregation in PdAu(1 11 1) alloy: Energetic and electronic DFT analysis, Chem. Phys. Lett. 503 (2011) 97-100. doi:10.1016/j.cplett.2010.12.064.

[42] C. Kwon, J. Kang, W. Kang, D. Kwak, B. Han, First principles study of the thermodynamic and kinetic properties of $\mathrm{U}$ in an electrorefining system using molybdenum cathode and $\mathrm{LiCl}-\mathrm{KCl}$ eutectic molten salt, Electrochim. Acta. 195 (2016) 216-222. doi:10.1016/j.electacta.2016.02.123.

[43] G.W. Watson, R.P.K. Wells, D.J. Willock, G.J. Hutchings, A comparison of the adsorption and diffusion of hydrogen on the (111) surface of Ni, Pd, and Pt from DFT calculations, J. Phys. Chem. B. 105 (2001) 4889-4894.

[44] J. Wellendorff, T.L. Silbaugh, D. Garcia-Pintos, J.K. Nørskov, T. Bligaard, F. Studt, C.T. Campbell, A benchmark database for adsorption bond energies to transition metal surfaces and comparison to selected DFT functionals, Surf. Sci. 640 (2015) 36-44. doi:10.1016/j.susc.2015.03.023.

[45] M. Naffati, S. Zanna, P. Cornette, D. Costa, P. Marcus, M.M. Abderrabba, S. Somrani, Adsorption of phenyl phosphate on Ni-Cr alloy surface: Experimental and theoretical investigations, Phosphorus, Sulfur Silicon Relat. Elem. 193 (2018) 185-191. doi:10.1080/10426507.2017.1395877. 
[46] C.L. Ren, H. Han, W. Bin Gong, C. Bin Wang, W. Zhang, C. Cheng, P. Huai, Z.Y. Zhu, Adsorption and diffusion of fluorine on $\mathrm{Cr}$-doped Ni(111) surface: Fluorine-induced initial corrosion of non-passivated Ni-based alloy, J. Nucl. Mater. 478 (2016) 295-302. doi:10.1016/j.jnucmat.2016.06.027.

[47] T. V. Pavlova, B. V. Andryushechkin, G.M. Zhidomirov, First-Principle Study of Adsorption and Desorption of Chlorine on $\mathrm{Cu}(111)$ Surface: Does Chlorine or Copper Chloride Desorb?, J. Phys. Chem. C. 120 (2016) 2829-2836. doi:10.1021/acs.jpcc.5b11794.

[48] S.H. Ma, Z.Y. Jiao, Z.X. Yang, Coverage effects on the adsorption of sulfur on Co(0 000 1): A DFT study, Surf. Sci. 604 (2010) 817-823. doi:10.1016/j.susc.2010.02.006.

[49] D.G. Sangiovanni, G.K. Gueorguiev, A. Kakanakova-Georgieva, Ab initio molecular dynamics of atomic-scale surface reactions: Insights into metal organic chemical vapor deposition of AlN on graphene, Phys. Chem. Chem. Phys. 20 (2018) 17751-17761. doi:10.1039/c8cp02786b.

[50] M. Stan, J.C. Ramirez, P. Cristea, S.Y. Hu, C. Deo, B.P. Uberuaga, S. Srivilliputhur, S.P. Rudin, J.M. Wills, Models and simulations of nuclear fuel materials properties, 445 (2007) 415-423. doi:10.1016/j.jallcom.2007.01.102.

[51] J.C. Chen, B. Reischl, P. Spijker, N. Holmberg, K. Laasonen, A.S. Foster, Ab initio Kinetic Monte Carlo simulations of dissolution at the NaCl-water interface, Phys. Chem. Chem. Phys. 16 (2014) 22545-22554. doi:10.1039/c4cp02375g.

[52] J.R. Boes, J.R. Kitchin, Modelling Segregation on AuPd(111) Surfaces with Density Functional Theory and Monte Carlo Simulations, J. Phys. Chem. C. 121 (2017) 3479-3487. doi:10.1021/acs.jpcc.6b12752. 